\title{
Control Methods in Data-Storage Systems
}

\author{
Giovanni Cherubini, Fellow, IEEE, Chung Choo Chung, Member, IEEE, William C. Messner, Senior Member, IEEE, \\ and S. O. Reza Moheimani, Fellow, IEEE
}

\begin{abstract}
The recording performance of data-storage devices, in which write/read elements move relative to a storage medium to reliably store and retrieve information, depends on the capability of servo mechanisms to provide the necessary positioning accuracy. The desired characteristics of servo mechanisms for datastorage systems include robustness against variations of environmental parameters, high resolution, accuracy, stability, and fast response. This paper presents a comprehensive overview of advanced servo-control methods for data storage. The applications are to well-established recording technologies, including magnetic tape and magnetic disk systems as well as CD/DVD/Blue-Ray optical data-storage systems. Moreover, newer holographic and nearfield optical systems and the emerging probe-storage technology are also addressed. Emphasis is given to the potential exhibited by the technologies considered for achieving ultra-high storage capacity, as required by the exploding demand in data-storage capacity for archival systems and massive multimedia data storage.
\end{abstract}

Index Terms-Control systems, hard-disk drive, holographic data storage, optical-disk drive, scanning-probe data storage, servomechanism, tape drive.

\section{INTRODUCTION}

$\mathbf{S}$ ERVO control systems are essential to achieve high throughput and near-optimum capacity in data-storage devices in which accurate positioning of write/read elements is required to reliably store and retrieve information. In today's increasingly interconnected world, there is an exploding demand in data-storage capacity for archival as well as real-time applications, as a staggering volume of digital data is being produced at an ever increasing pace. According to an International Data Corporation study [1], the amount of digital information produced in 2011 is expected to approach 1.8 zettabytes, corresponding to $1.8 \times 10^{21}$ bytes, or 10 times that produced in 2006, with a compound annual growth rate of about $60 \%$, i.e., significantly faster than the growth rate of storage capacity. Furthermore, new regulatory requirements demand that a larger fraction of this data be preserved, for example, in the government and healthcare sectors.

Manuscript received December 05, 2010; revised October 24, 2011; accepted November 05, 2011. Manuscript received in final form November 15, 2011. Date of publication December 13, 2011; date of current version February 01, 2012. Recommended by Associate Editor G. Guo.

G. Cherubini is with IBM Research-Zurich, 8803 Rüschlikon, Switzerland (e-mail: cbi@zurich.ibm.com).

C. C. Chung is with the Division of Electrical and Biomedical Engineering, Hanyang University, Seoul 133-791, Korea.

W. C. Messner is with the Department of Mechanical Engineering, Carnegie Mellon University, Pittsburg, PA 15213 USA.

S. O. R. Moheimani is with the School of Electrical Engineering and Computer Science, The University of Newcastle, Callaghan NSW 2308, Australia.

Color versions of one or more of the figures in this paper are available online at http://ieeexplore.ieee.org.

Digital Object Identifier 10.1109/TCST.2011.2176942
Servo control is becoming increasingly important for systems such as hard-disk drives (HDDs), tape drives, and optical disk drives (ODDs). Significant new technologies are emerging in this field in response to challenging requirements for future storage systems. Examples are described in the programs of the Information Storage Industry Consortium (INSIC), which are aimed at achieving extremely high-density recording in HDDs, on the order of a few $\mathrm{Tb} / \mathrm{in}^{2}$, and extremely high tape capacities, on the order of a few tens of terabytes, within the next decade. The INSIC also envisages significantly higher capacities for ODDs, up to the terabyte range, that can be achieved by resorting to near-field recording.

For an overview of nanopositioning technologies and devices emphasizing the key role of advanced control techniques in improving precision, accuracy, and speed of operation of these systems in various applications, the reader is referred to [2].

To our knowledge, the present paper provides the first comprehensive survey of control techniques that find application in storage technologies. Advanced servo-control design methods are reviewed, with emphasis on those elements that are considered essential for achieving ultra-high storage capacities. For example, in HDDs, robust control systems for dual-stage servo actuators are being considered to perform fine head positioning [3], [4]. Feedback-control systems for nanoprobe-based heating in heat-assisted magnetic recording are envisaged to control the nanoscale gap between the probe and the recording medium [5]. In tape drives, new control methods are needed to compensate the dynamic skew of the head relative to the moving tape by means of tilting rollers and/or actuators with more than one degree of freedom [6]. High-bandwidth piezoelectric actuators are considered for enhancing the performance of track-following loops in the presence of high-frequency lateral tape motion (LTM) [7]. In ODDs, near-field recording aims at reducing the size of the beam spot by increasing the numerical aperture of a focusing lens using solid immersion lens technology [8], [9]. When using a blue laser diode as light source, the gap distance should be controlled to less than $100 \mathrm{~nm}$, with tight margins. Stable servo systems are needed to prevent collisions between the lens and the disk surface and to achieve good system performance [10].

Emerging data-storage systems are also addressed that will inherently require high-performance position control. Holographic data storage holds the potential for a volumetric density greater than $100 \mathrm{~Gb} / \mathrm{cm}^{3}$ and throughput larger than 1 $\mathrm{Gb} / \mathrm{s}$ [11]. The tremendous potential of scanning-probe storage devices to achieve ultra-high areal density has also been recognized [12]-[15]. In these devices, nanopositioning techniques to navigate a scanner over the storage medium with nanometer accuracy play a fundamental role [16], [17]. Recent advances 
have demonstrated the feasibility of achieving densities of more than $2 \mathrm{~Tb} / \mathrm{in}^{2}$ with scanning-probe storage technologies [18]. Such applications promise a dramatic increase in the achievable capacity of storage devices for archival systems and multimedia data storage.

This paper is organized as follows. An overview of controlrelated research in tape drives is presented in Section II. Servocontrol methods for HDDs are discussed in Section III. The general ODD architecture and a survey of related control issues and trends are presented in Section IV. Control techniques for holographic data storage are addressed in Section V. Finally, a review of advances in nanopositioning for probe storage is provided in Section VI.

\section{TAPE DRIVES}

The first commercially available data-storage device relying on magnetic recording was the IBM 726 tape system introduced in 1952 [19], [20]. The 20-inch-diameter cartridge with a 720 meter-long magnetic tape had a capacity of 1.4 MB. The computations required by the control algorithms were performed in the same computing unit that used the tape for external data storage. Only in 1956 was a dedicated control unit finally included in the drive.

Since 1952, the volumetric density of data recorded on tape has increased about ten-million-fold, leading to a dramatic reduction in storage cost per gigabyte. State-of-the-art magnetic tape cartridges can currently store $4 \mathrm{~TB}$ of uncompressed user data by means of linear tape recording, whereby data is recorded in long parallel tracks that extend over the entire length of the tape. Linear serpentine recording, a variation of linear recording that allows tracks to be shingled in a serpentine manner to increase track density, has been standardized in the linear tape open (LTO) format [21], [22].

The main driving force of the progress in volumetric density of magnetic tape has been the areal density increase resulting from enhancements in linear density and track density. In the past, the linear density increased much more strongly than the track density for tape systems [23], [24]. An increase in linear density translates into a smaller distance between adjacent bit cells, leading to an increase in inter-symbol interference. Powerful data-detection and error-correction coding techniques have been introduced to cope with the inherent variability and the disturbances that characterize the read channel [25], [26]. On the other hand, a higher track density implies a narrower track width and narrower write/read heads, leading to losses in the signal-to-noise ratio (SNR) and possibly to inter-track interference [6].

High-performance magnetic tape media are expected to play an important role in future tape storage systems, as indicated by the recent demonstration of $29.5 \mathrm{~Gb} / \mathrm{in}^{2}$ recording areal density by IBM in collaboration with Fujifilm [27]. This result was obtained by introducing advanced system concepts as well as a new magnetic tape based on ultra-fine, perpendicularly oriented barium-ferrite $(\mathrm{BaFe})$ particles, to replace the metal-particulate (MP)-type media currently used in linear tape recording technology. In the remaining part of this section, advances in tape

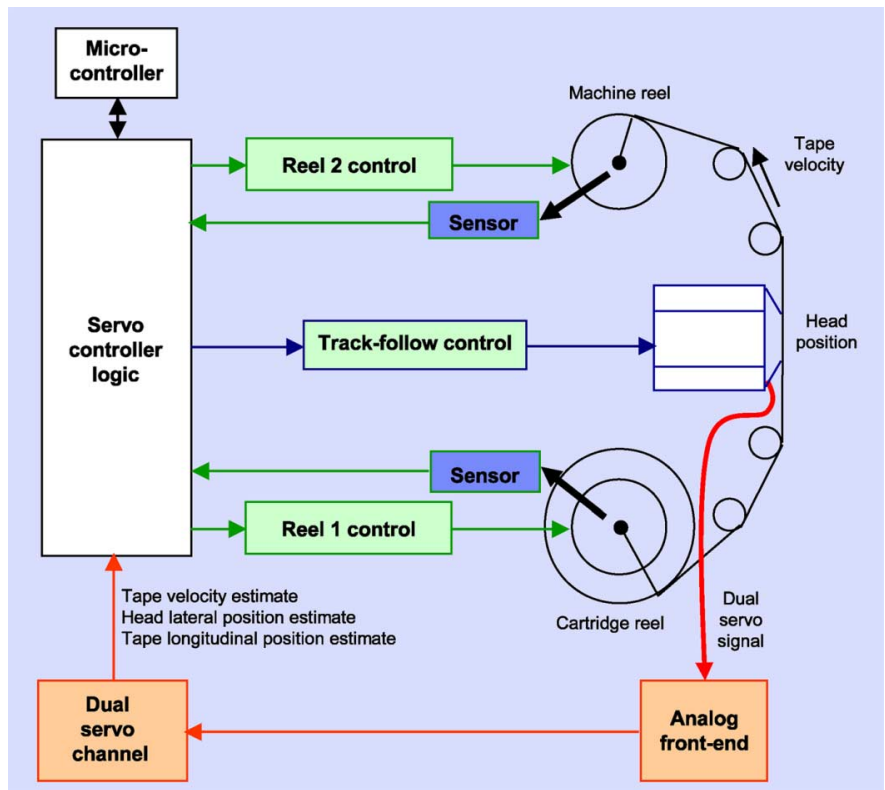

Fig. 1. Block diagram of reel-to-reel and track-following servo using a dual servo channel.

transport and track-following servo systems will be discussed, which will enable substantial further progress in track density.

\section{A. Tape Path}

The block diagram of a tape-drive system is shown in Fig. 1. In tape drives, rolling elements transport the tape between the cartridge reel and the machine reel over the magnetic read/write head. Traditionally, flanged rollers have been used in the tape path to constrain LTM, which represents one of the main impairments limiting the maximum track density. Sources of LTM include reel motor vibrations, tape tension transients stemming from torque variations in the reel motors, run-out of tape reels and rollers, tape wear, and contact between the tape edge and a flange of a roller or a reel [28]-[32].

An important tape-path design goal is the prevention of debris accumulation on the roller flanges due to their contact with the tape edges. This might cause LTM disturbances that often exceed the bandwidth of the track-following actuator, which is on the order of $1 \mathrm{kHz}$ in state-of-the-art drives. One approach is to change the roller layout or to remove the flanges from the rollers. In this manner, the high-frequency content of the LTM disturbance can be significantly reduced, however at the expense of introducing other challenges [6]. First, the range of lateral tape displacement may become larger. Second, dynamic skew, which arises whenever the gap of the head does not remain perfectly perpendicular to the direction of longitudinal tape motion, may also attain large values. Dynamic skew tends to induce increasing loss of signal as the frequency rises, as well as readback-signal frequency fluctuations, which are difficult to track by the phase-lock loops usually implemented in the data channels [33]. These additional challenges may be addressed by resorting to an advanced actuator that is capable of following an LTM with large excursions and of servoing its rotation angle to keep the head perpendicular to the direction of longitudinal tape motion. 


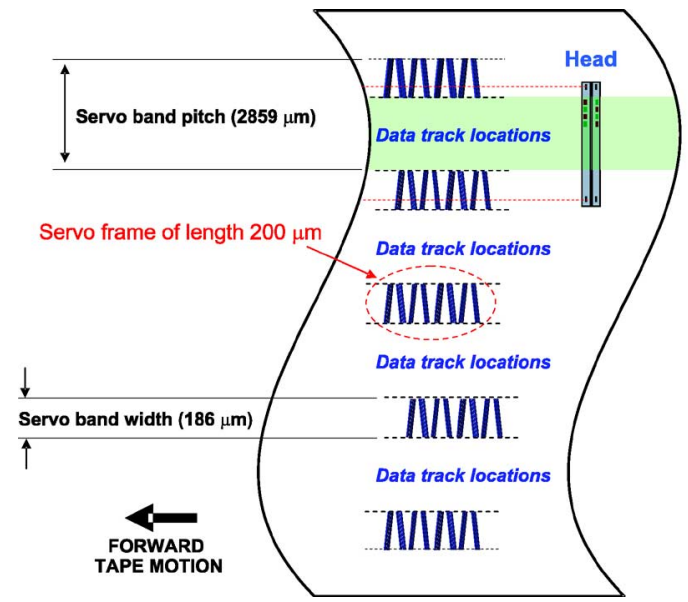

Servo band 0
Data band 3
Servo band 1
Data band 1
Servo band 2
Data band 0
Servo band 3
Data band 2
Servo band 4

Fig. 2. Layout of a 1/2-inch tape according to the LTO standard.

\section{B. Timing-Based Servo and Synchronous Servo Channel}

In tape drives, the core of the reel-to-reel and track-following servomechanisms is the system that generates the feedback information for the servo controllers. In the so-called timing-based servo (TBS) system [34] adopted in today's LTO drives, five dedicated servo bands are written on the 1/2-inch tape during manufacturing. Thus five servo bands straddle four data bands, in which user data are stored on several written tracks, as illustrated in Fig. 2. The recorded servo patterns consist of transitions with two different azimuthal angles. The lateral position of the narrow head element reading the servo patterns and the tape velocity are estimated from the relative timing of the pulses in the servo bursts. Furthermore, information indicating the longitudinal position (LPOS) of the head along the tape is encoded into the servo frames using pulse-position modulation. The head lateral position and tape velocity estimates and the longitudinal position information are obtained by a digital servo channel processing the samples of the readback servo signal. The sequence of lateral position estimates is used to generate a position-error signal (PES) that is fed back to the track-following servo, whereas the tape velocity estimates and the longitudinal position information are fed back to the reel-to-reel servo. A model of the readback servo signal to analyze the impact of the various TBS elements on the overall servo performance has been developed in [35] and [36].

To achieve high-performance servo control, it is essential that the measurement noise affecting the estimates be minimized. A synchronous servo channel architecture as shown in Fig. 3 allows near-optimum filtering of the readback servo signal, minimizing measurement noise [37]. The digital interpolator/correlator implemented in a synchronous servo channel achieves a close approximation of the optimum filter for the detection of the readback servo signal independently of tape velocity. It yields a substantially improved quality of the servo parameter estimates compared to the direct observation of the peak arrival times of the readback servo signal. Two servo readers are normally active in a head module, from which a dual servo signal is derived, see Fig. 1. Therefore, a dual synchronous servo channel including signal-combining techniques can be used to further improve the system performance [38].

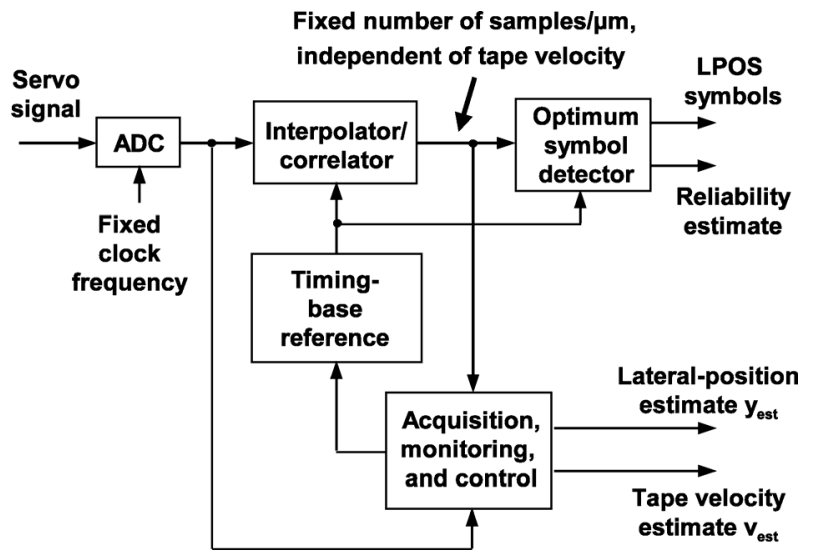

Fig. 3. Block diagram of a synchronous servo channel.

\section{Tape Transport}

The reel-to-reel and track-following servo systems are of fundamental importance to guarantee best read-channel performance on all parallel data channels during tape drive operation. In particular, tight control of the tape tension and velocity is necessary for introducing thinner media and mitigating lateral tape motion, thereby also enabling an increase in volumetric density. Fig. 1 shows the block diagram of a tape drive using conventional reel-to-reel and track-following servo systems. As the two reels are driven independently to control tape tension and velocity, the reel-to-reel system is inherently a multi-input, multi-output (MIMO) system [39]. As mentioned above, a dual servo channel provides estimates of the tape velocity, tape longitudinal position, and head lateral position. In addition, Hall sensors may be used to obtain tape velocity information prior to initial parameter acquisition by the servo channel.

The tape-transport problem is related to the transport of webs, as found for example in the manufacture of paper, plastic, and sheet metal. Early work on the dynamic analysis and control of the lateral and longitudinal displacements of a moving web is discussed in [40] and [41]. A model of the tape in a reel-to-reel system is illustrated in Fig. 4, where $K_{T}$ and $D_{T}$ denote the spring constant and the damper coefficient of the tape, and $R_{1}$ and $R_{2}$ the radii of the cartridge reel and the machine reel, respectively. Because the radii and inertia of the reels vary slowly during transport, and air entrainment dynamics rapidly and nonlinearly change the spring constants of the system, the dynamics of web transport are both time-varying and nonlinear. A statespace formulation of the MIMO reel-to-reel system with a controller design based on the sequential loop closing (SLC) technique, and tension feedback from strain gauge sensors located in the tape path, is presented in [42]. A control system design obtained via the linear quadratic regulator (LQR) technique, in which a gain-scheduling approach is introduced to cope with the time-varying system dynamics, is proposed in [43].

The phenomenon of air entrainment, whereby friction draws a thin layer of air into the take-up reel, causing several layers of tape to be wound loosely, has been studied extensively both in steady state [44], [45] and during transients [46]. As the tape velocity increases, air entrainment increases the effective length of 


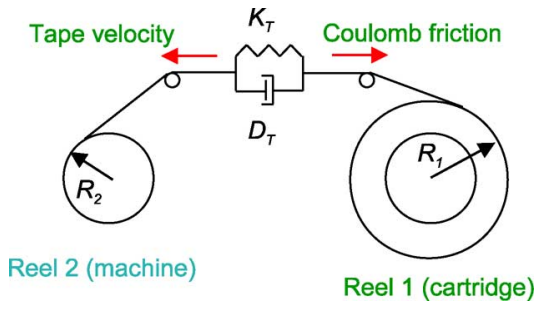

Fig. 4. Model of tape in a reel-to-reel system.

the tape path, thus lowering the spring constant of the tape and hence the resonance frequency of the transport system. Designs of closed-loop controllers for reel-to-reel systems taking into account air entrainment exist that are based on the gain-scheduling approach, with a 2-D grid to cover the desired range of tape velocity and tension [47], $H_{\infty}$ techniques [48], and guaranteed robustness properties [49]. Open-loop time-optimal control in the presence of air entrainment is addressed in [50].

The closed-loop controllers discussed above rely on straingauge sensors placed in the tape path to provide tension feedback. In commercially available tape drives, however, the use of tension transducers often is not desirable for cost reasons. The problem of robust control of tape transport systems using a tension observer instead of tension sensors has been addressed in [51]-[53].

A further impairment affecting the performance of reel-to-reel servo systems and the quality of readback signals in data channels is reel eccentricities that induce undesired tape tension ripples around the nominal tension value during cruise velocity. These tension ripples are quasi-periodic disturbances with slowly varying frequencies in the range from 10 to $60 \mathrm{~Hz}$, depending on the reel radii and tape tangential velocities. In tape transport, this problem is particularly serious when the reel rotation frequencies are near the resonance frequency determined by the tape path. Adaptive control algorithms for the suppression of time-varying once-around disturbances are presented in [54].

The pulsating electromagnetic torque produced by the brushless DC motors, also known as permanent magnet synchronous motors, which are commonly used as reel drivers in tape transport, constitutes a further source of tension ripples. Pulsating torque components are often generated by the interaction of stator current magnetomotive forces and rotor electromagnetic properties [55], [56]. Various algorithms for torque ripple attenuation in brushless DC motors are proposed in [57]-[60].

\section{Track Following}

The main task of the track-following controller is to position the head actuator as accurately as possible during write/read operations in the presence of LTM and other disturbances [61]. The $3 \sigma$ (three standard deviation) value of the PES represents an estimate of the maximum amplitude of the deviation from the head lateral target position during track following. To avoid read and write errors, which are usually referred to as track misregistration (TMR), the largest PES amplitude is kept below $10 \%$ of the track width in commercial drives. In a linear control scheme, the closed-loop transfer functions determine the track-follow performance in terms of disturbance rejection capabilities and sensitivity to measurement noise. The disturbance rejection transfer function can be approximated as a high-pass filtering function with a cutoff frequency that roughly corresponds to the control bandwidth. In [62], system identification methods are used to estimate a high-order filter that accurately models the dynamics of the disturbance rejection transfer function and the spectral content of additional disturbances that contribute to the PES, besides the LTM. Using the estimated model in conjunction with LTM measurements obtained by optical sensors, the PES can be accurately predicted and the impact of additional disturbances, e.g., servo pattern written-in errors occurring during tape manufacturing, can be evaluated independently of LTM.

Note that increasing the bandwidth of the track-following system leads to a higher sensitivity to measurement noise. Furthermore, observing Bode's sensitivity integral theorem, a frequency interval exists in which the position error is amplified by the controller. Therefore knowledge of the frequency characteristics of the various disturbances is necessary to optimize the closed-loop system performance. From a spectral characterization of the LTM, several quasi-periodic components can be identified. A linear quadratic Gaussian (LQG) architecture for disturbance suppression, whereby the estimator is augmented with the states of virtual systems modeling the quasi-periodic disturbance sources, is presented in [63]. A method based on real-time LTM measurements and the double-Youla parameterization to design a robust controller that is tunable to any drive-cartridge pair is illustrated in [64] and [65].

Tape-stacking irregularities in the supply reel, e.g., stack shifts or staggered wraps, lead to LTM events that are characterized by a large amplitude and low frequency. To effectively mitigate the effects of such events, the track-following system can be enhanced by a feed-forward control architecture using information on the LTM captured by an optical sensor sensing the later tape-edge displacement. The sensor is placed in the tape path at a certain distance from the head. As LTM disturbances propagate along the tape path, the LTM measured by the sensor will affect the track-following process with a delay that depends on the sensor-head distance and the tape velocity. This LTM information can be used by a feed-forward controller to improve system performance, as done in the track-following architecture combining feed-forward and feedback control also presented in [63].

An active tape-guiding technique is proposed in [66] to mitigate the effects of flangeless rollers in the tape path. The method resorts to controlling the tilt of one or more rolling elements based on the measured LTM. The active tape-guiding mechanism has been characterized in terms of the lateral tape displacement versus the input current to the tilting roller actuator at different tape velocities, and the closed-loop performance has been evaluated.

The limits of a track-following servo based on the TBS system and a voice-coil motor (VCM) actuator are investigated in [67], where a PES standard deviation of $15 \mathrm{~nm}$ is demonstrated. The nanoscale positioning accuracy shown in [67] is achieved by introducing advanced concepts for several system elements, namely, a tape path with flangeless rollers to mitigate 
high-frequency LTM components, an $H_{\infty}$ track-following controller, and a new geometry of the servo patterns in the TBS system in conjunction with a synchronous servo channel to minimize the PES measurement error.

To increase the open-loop bandwidth of the track-following system, a piezo-actuator has been considered in [68]. A dualstage actuator head has been proposed in [69], similarly to the approach introduced in HDDs. The main difference, however, is that a tape drive head design requires a linear actuator, whereas HDD designs resort to rotary actuators. The dual-stage linear actuator is based on a VCM that acts as a primary "coarse" actuator with large strokes, following low-frequency LTM, i.e., less than $1 \mathrm{kHz}$, and on a stacked piezoelectric (PZT) stage that acts as a secondary "fine" actuator with small strokes, compensating for high-frequency LTM. The controller design for the resulting dual-input, single-output (DISO) system is presented in [7]. The dual-stage controller is based on the so-called PQ method, which allows the decomposition of the DISO system into two single-input, single-output (SISO) systems, and addresses the coupling between the actuators by frequency separation [70].

In state-of-the-art drives, the lateral position of the head is controlled with a VCM, usually represented by a mass-spring-damper model. The overall head-maneuvering system, however, is fairly complex. The flexible modes of the system can cause nonnegligible resonances and impair system performance, e.g., by increasing the time required for the head to settle on a desired track. The application of feed-forward input shaping to head control to reduce the settling time in the presence of vibrations is illustrated in [71].

The initial positioning of the head, which aims at properly aligning two servo readers over two adjacent servo bands, see Fig. 2, can be regarded as a moving target problem if the servo readers alignment occurs in the presence of large LTM. A solution to this moving target problem is proposed in [72], where not only the servo readers, but also the data readers in the head are used to detect the presence of a valid, albeit distorted, servo signal. The distance between the servo reader and the servo band is continuously estimated by identifying the data reader detecting the presence of a servo signal. This information is then provided to a control element that determines a trajectory for the head and allows the servo readers to rapidly land over the servo bands.

\section{E. Dynamic Skew Compensation}

As mentioned earlier, it may be desirable to differently shape or completely remove the flanges from the rollers in the tape path to mitigate high-frequency LTM. The low-frequency LTM, however, may become larger, potentially leading to large tape-to-head skew, as illustrated in Fig. 5(a), in which the head and the tape alignment are shown in the presence of a skew angle $\theta$. The head usually includes two modules that are placed face to face to provide bidirectional read-after-write capability, which is needed to guarantee that no loss of data occurs during write operations [20]. Each module hosts pairs of data writers and readers that are spanned by two servo readers for track following, as shown in Fig. 5(b). By observing the head geometry, it is clear that a skew between tape and head module may

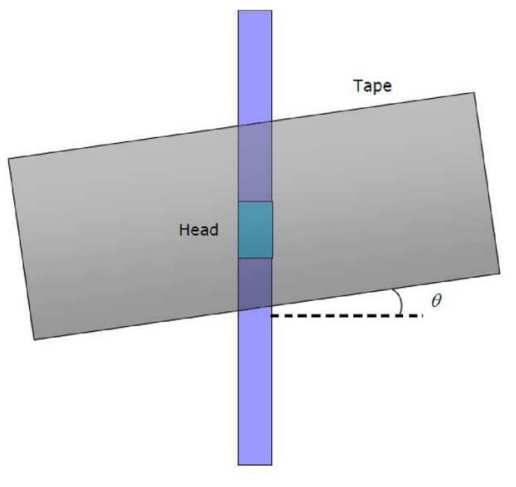

(a)

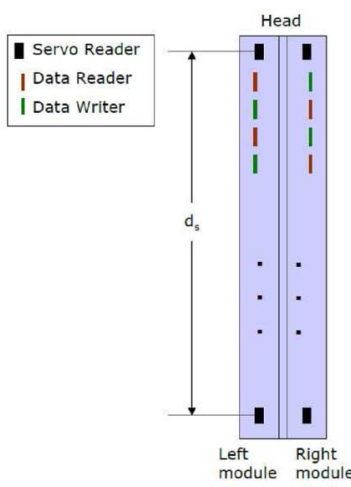

(b)
Fig. 5. Illustration of (a) skew and (b) head modules in a tape system.

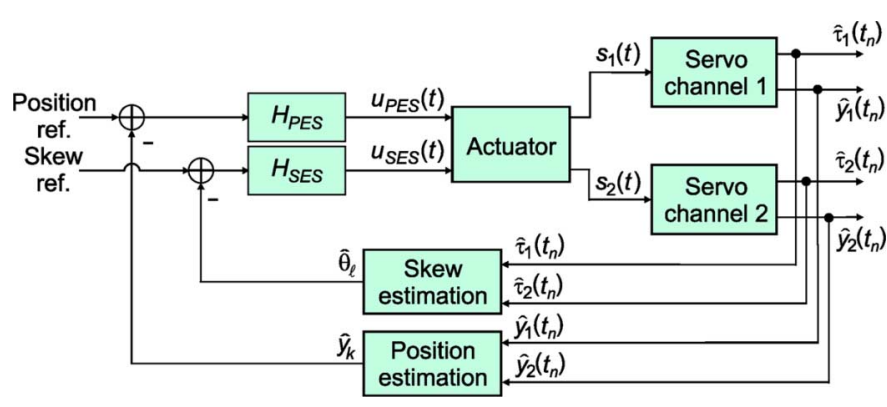

Fig. 6. Block diagram of joint track-following and skew-compensation servo.

significantly reduce the margins for reliable read-after-write operation. Compensation with skew-following actuation might thus be required to keep the head perpendicular to the direction of longitudinal tape motion. For this purpose, an actuator may be used that includes a rotational as well as a translational degree of freedom [6].

A control system for the joint track-following and skew-compensation servo is illustrated in Fig. 6 [73]. An estimate of the skew-error signal (SES) can be obtained by measuring the time intervals between peaks of corresponding pulses in the bursts of servo signals from the top and bottom active servo readers, where it is assumed that the servo patterns in adjacent servo bands are perfectly aligned. The measure of the shift between the servo patterns on the servo channels is given by

$$
\xi=\hat{v}\left(\tau_{2}-\tau_{1}\right)
$$

where $\hat{v}$ denotes the tape velocity estimate, and $\tau_{1}$ and $\tau_{2}$ denote the time instants at which the signal peaks are detected on channel 1 and 2, respectively. Considering the case of nonzero displacement between servo patterns on adjacent channels, as for example in the LTO format [21], an estimate of the skew error is then given by

$$
\hat{\theta}=\arctan \left(\frac{\xi-\xi_{\text {ref }}}{d_{s}}\right)
$$

where the reference shift $\xi_{\text {ref }}$ depends on the servo bands spanned by the head module, and $d_{s}$ denotes the distance between the top and the bottom servo reader. 


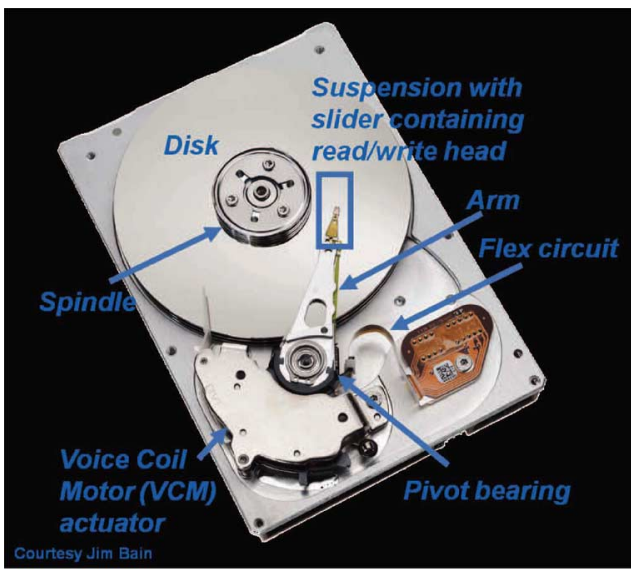

(a)

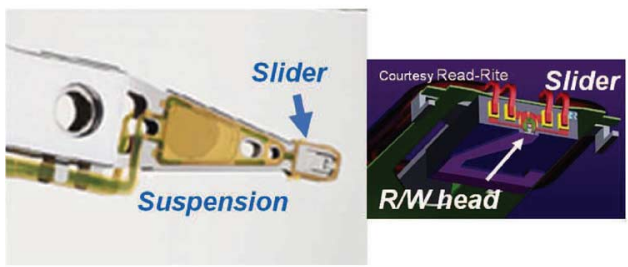

(b)

Fig. 7. Disk drive photos and schematic with key components labeled. (a) Disk drive. (b) Suspension and slider.

\section{F. Summary and Outlook}

Tape systems will continue to play a key role in the protection and preservation of data for many businesses and organizations, providing the capability to read data back from tape reliably and without errors whenever it is needed, even after several years. Moreover, tape remains an integral part of a tiered storage infrastructure, especially because of its moderate total cost of ownership. As inactive data does not consume energy, the favorable impact on the power budget of IT organizations makes tape storage an inherently "green" technology, and hence increasingly attractive for both archival and enterprise applications.

Tape cartridge capacities will keep growing at about $40 \%$ compound annual growth rate, requiring further advances in tape drive technologies. The push towards increasing areal densities will lead to much higher track densities, with the requirement of highly sophisticated track-following servo to achieve nanoscale positioning performance over flexible tape media. To maintain the pace of increase in volumetric density, thinner media will be adopted, requiring tight tension control in the reel-to-reel servo. In the years to come, tape storage will continue to present servo engineers with challenging tasks and with the need for innovations to sustain the further evolution of this six-decade-young technology.

\section{HARD-DISK DRIVES}

Magnetic hard-disk drives, the primary device for near-term nonvolatile storage for computer systems, are fascinating mechatronic devices featuring high-speed nanometer-scale positioning, see Fig. 7. They are being produced in very large volumes, hundreds of millions of units per year, at low cost.
Since IBM introduced the first disk drive in 1956, the random access memory accounting (RAMAC) system, with a capacity of $5 \mathrm{MB}$, capacities have increased by a factor of more than 200000 to over 1 TB per drive. The cost per bit has decreased by even greater factors. Commercial disk drives are routinely available for as little as USD 0.10 per GB, 300 million times less than the RAMAC, in which the storage cost was USD 32000 per MB in 1956 dollars.

Areal storage densities have increased by similarly large factors: from the RAMAC density of approximately 20 bits per $\mathrm{mm}^{2}\left(1.2 \mathrm{~kb} / \mathrm{in}^{2}\right)$ to over $4 \times 10^{8}$ bits per $\mathrm{mm}^{2}\left(250 \mathrm{~Gb} / \mathrm{in}^{2}\right)$, a factor of more than 200 million, allowing drastic decreases in size. The disks in the RAMAC were $2 \mathrm{ft}(0.6 \mathrm{~m})$ in diameter, and the entire system had a mass of well over $1000 \mathrm{~kg}$. Although disk drives with disk diameters of $22 \mathrm{~mm}$ ( 0.85 in) have been produced, the preferred form factor currently is approximately $65 \mathrm{~mm}$ (2.5 in), with a mass of a few hundred grams.

In spite of the impressive and continuing reductions in cost and size, there is great concern that solid-state memory will replace disk drives in the near future. This concern is misplaced. The reason is the total capacity all disk drives shipped compared with the potential total capacity of solid-state memory. Even if the entire semiconductor industry were devoted to producing such memory, the total shipped capacity of disk drives would be about 40 times larger than the potential shipped capacity of solid-state memory. Given the multi-billion-dollar cost of building new semiconductor fabrication plants, Fontana and Hetzler estimate that replacing disk drives with solid-state memory would require an investment of USD 1 trillion [74].

The advantage of solid-state memory over disk storage is in the so-called "cost of the first gigabyte." Simply put, one can buy a single gigabyte of solid state memory, but the cost of $1 \mathrm{~GB}$ of disk storage is the cost of an entire drive, which is that for 50 or more gigabytes. For large storage capacities, disk storage is much cheaper than solid-state memory, but for a few gigabytes, solid-state memory is much cheaper and much smaller. Thus solid-state memory has completely replaced disk storage in consumer applications requiring only a few gigabytes of memory, e.g., cameras and mobile phones.

\section{A. Operation and Mechanical Specifications}

HDDs, or simply "disk drives", store data as magnetic patterns perpendicular to the plane of spinning disks made of glass or aluminum. These disks are coated with a complex laminate of nanoscale materials which can change their magnetization (the recording layer), propagate magnetic fields (the "soft underlayer"), or are used to provide dimensional matching between the lattice structures of two different layers. The top of these recording layers is protected with a layer of diamondlike carbon (DLC), and the surface of the DLC is covered with lubricant.

The data bits of a disk drive are approximately rectangular and arranged in concentric tracks. The data bits are 4-8 times wider in the radial direction than in the tangential direction. At a bit aspect ratio of $4: 1$ and an areal density of $4 \times 10^{8}$ bits per $\mathrm{mm}^{2}$, the track density is approximately $10^{4}$ tracks per $\mathrm{mm}$ $\left(250 \times 10^{3}\right.$ tracks per inch), and the linear density in the tangential direction is $4 \times 10^{4}$ bits per $\mathrm{mm}\left(10^{6}\right.$ bits per inch). The track 
pitch (the distance from the center of one track to the center of the adjacent track) is approximately $100 \mathrm{~nm}$, with profound implications for the head-positioning control system.

The data are read and written by two separate transducers (collectively called the "read/write head" or "W/R head") constructed on the trailing edge of a small, intricately contoured composite ceramic block of aluminum oxide and titanium carbide (ALTIC) called the slider. The slider is about $1 \mathrm{~mm} \times 0.5$ $\mathrm{mm} \times 0.2 \mathrm{~mm}$, and it rides on a self-generated air bearing, i.e., it "flies". The distance from the read and write transducers to the disk surface is the "fly height." Proper functioning at today's data-storage densities requires that the fly height be about $6 \mathrm{~nm}$, because the magnetic signal from the disk decays exponentially with distance. The fly height is maintained even as the skew angle of the slider and the relative speed between the slider and the rotating disk change significantly and even as the servo system moves the $\mathrm{W} / \mathrm{R}$ head from the inner edge (inner diameter or ID) to the outer edge of the disk (outer diameter or OD). A favorite analogy in the disk-drive industry is that the fly height and size of the slider and the speed of rotating disks are dimensionally analogous to a Boeing 747 flying at an altitude of few millimeters at thousands of meters per second.

The write transducer is a very small, electromagnetic element constructed using thin-film processing technologies. It has a narrow leading pole for concentrating the magnetic field of the head to change (write) the magnetization of the recording layer of the material. The trailing edge is much longer to complete the magnetic circuit through the soft underlayer of the disk without concentrating the magnetic fields to overwrite data that has already been recorded. The trailing pole is trimmed to be trapezoidal so that it does not overlap adjacent tracks when the head is skewed.

The read transducer is a structure that relies on the giant magneto-resistive (GMR) effect. The resistance of the GMR material of the read transducer changes significantly in response to a change in magnetic field. The change in the resistance results in a voltage signal of a few millivolts, which the read channel electronics of the disk drive can amplify and demodulate.

Depending on the application, the spindle motor rotates the disks at anywhere from $3600 \mathrm{rpm}(60 \mathrm{~Hz})$ to $10000 \mathrm{rpm}(167$ $\mathrm{Hz})$, and drives with $15000 \mathrm{rpm}(250 \mathrm{~Hz})$ have even been developed. Typically, the highest spindle speeds are found in enterprise drives, where low latency (the time to reach the location of the data on the disk) is at a premium and acoustic noise is less of an issue. The lowest speeds are found in disk drives for laptops, where requirements for low power dominate the desire for low latency.

The slider is mounted on a head-gimbal assembly (HGA) which is itself part of the suspension of the disk drive. The suspension is a highly engineered stainless-steel leaf spring that holds the head against the disk with a precise preload. The HGA allows the slider to rotate slightly to maintain the correct orientation relative to the disk as the slider moves back and forth across the disk. The suspension is attached to the much stiffer arm, often called the "E-block," because from the side, it resembles the shape of a capital $\mathrm{E}$. The arm rotates about a pivot bearing, which is a small ball bearing.
The actuation for the arm is provided by the electromagnetic "voice coil actuator" (VCM), which is a flat coil of wires located between the poles of a flat, powerful rare-earth magnet. The signals to the VCM and the signals to the W/R heads of the disk drive pass through the flex circuit, which is a laminate of polymer and conductors.

To provide position information to the servo system, special sectors, called "servo sectors", are written at regular intervals around the disk. Typically these servo sectors occupy about $10 \%$ of the total disk area. These patterns are written onto the disk on special machines (servo writers) before the disks are mounted onto the spindle of the disk drive, or they are written by the disk drive itself during the burn-in and test process [75]. This latter process is called "self-servo write," and can take many hours to format hundreds of thousands of data tracks. In addition, as the write transducer is displaced longitudinally from the read sensor by a finite distance, the drive system must account for the skew of the slider relative to the track, which will cause the write transducer to be radially displaced by several tracks away from the read transducer.

One of the unique aspects of the servo system is that it is a true sampled-data control-system because position information is only available when the head passes over the servo sector. Changing the sample rate of the control system requires complete reformatting of the disks, and thus is impossible after manufacture.

Fig. 8 schematically depicts the architecture of a servo sector. The servo sector may contain other fields not shown, such as those for automatic gain compensation. The Gray code of the servo sector provides information on the track number. The "servo pattern" provides signals demodulated to determine the fine position of the head. The figure shows the so-called "A-B pattern" or "amplitude pattern", in which the relative amplitudes of the readback signal over the sub-fields of the pattern indicate the head position. Other patterns determining the position using the amplitude, phase, timing, or frequency of the readback signal have been devised [76].

\section{B. Servo System Functions and Challenges}

The servo system of a HDD has two main functions, namely, track seek, which is the rapid movement of the W/R head from one data track to another, and track following, which is the function of keeping the read or write transducer within acceptable limits of the center of the data track for reading or writing. Recall that the deviation of the head from the track center is the track misregistration and is usually reported as a $3 \sigma$ percentage of the track pitch, the nominal distance from the center of one track to that of the next. Each function has its own challenges, but both are complicated by the fact that the actuator is not co-located with the sensor. Consequently there are numerous mechanical resonances in the suspension, the actuator arm, the pivot bearing, and the voice coil. However, the dynamics of disk drives, although complex, are largely linear, and thus disk drives are candidates for control-design methodologies for linear systems. 


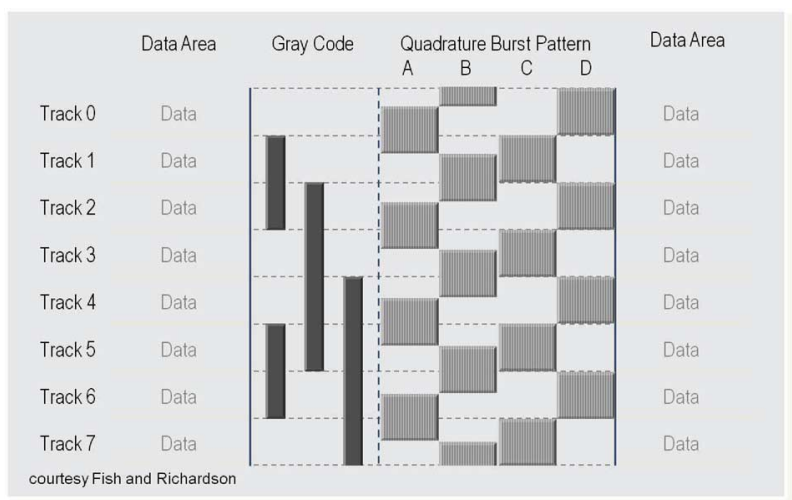

(a)

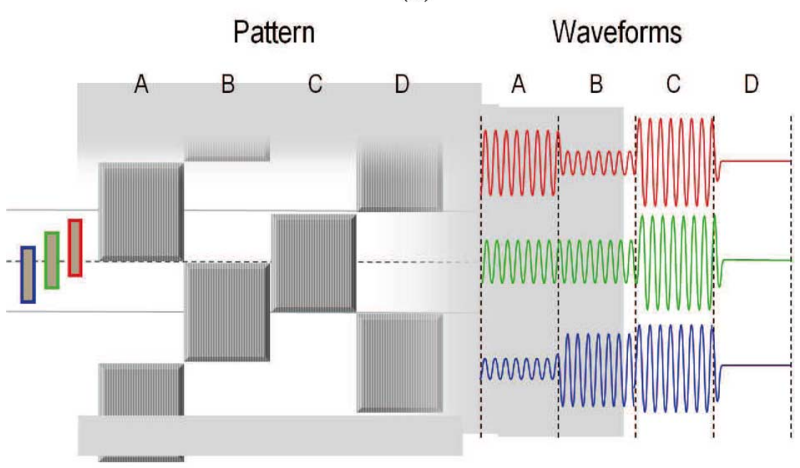

(b)

Fig. 8. Schematic depiction of (a) the servo sector and (b) amplitude pattern with read back signals from the head. The small rectangles in (b) represent the head at different cross-track positions. The colored waveforms correspond to the signals from the head position with that color.

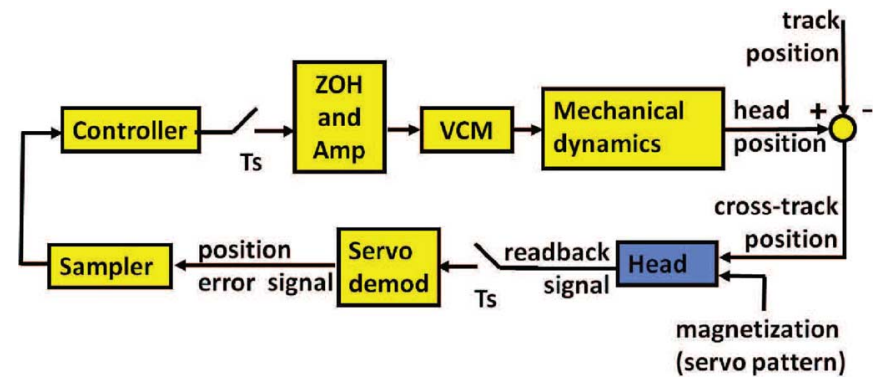

Fig. 9. Schematic of an HDD servo system.

Fig. 9 shows a functional block diagram of a servo system of a HDD. The relevant quantity for tracking is the cross-track position. Note that the actual track position enters as an output disturbance. The measured head position depends on the crosstrack position and the magnetization of the disk. Thus, any defects in the servo pattern become repeatable errors in the position signal during operation.

The track seek must balance the need for rapid movement with the need for low noise in consumer applications, e.g., desktop computers [77], low power consumption in mobile applications, e.g., laptops [78], and low emitted vibration due to reaction forces and moments in applications with multiple drives in a single rack, e.g., server systems [79]. The seek control must also account for saturation of the amplifier and prominent hysteresis nonlinearities in the friction of the pivot bearing [80]. There are a wide variety of short seek algorithms used in the industry, and variations of the venerable proximate time optimal servo (PTOS) [39], a modification of bang-bang control, remain a viable candidate for seek control in many cases.

The most important developments in recent years with respect to addressing these challenges are algorithms for seek scheduling that take the rotational latency into account. These algorithms coordinate the seek with the time needed for the rotation of the disk to bring the data to the head, allowing slower seeks in many instances. At the same time, there have been numerous reports of efforts to design feedforward and feedback control of the seek to reduce settling time, noise, power, and emitted vibrations [81]-[83].

With respect to tracking, improvements in the channel have somewhat relaxed the specifications for TMR for reading and writing. Nevertheless, the $3 \sigma$ specification for TMR is still on the order of $\pm 10 \%$ of the track pitch for reading and $\pm 5 \%$ for writing. Thus, the head-positioning system must keep the head to within 5-10 $\mathrm{nm}$ of the track center in the presence of numerous disturbances. Disk-drive designers continue to improve the mechanical dynamics, sensors, actuators, and control algorithms to reduce the effect of mechanical disturbances and noise on the drive while adhering to stringent cost constraints.

The disturbances that affect tracking are either persistent or intermittent. Sources of intermittent disturbances include external shocks and vibrations, vibrations from other HDDs in server arrays, and the occasional impact between the slider and the disk. Persistent disturbances are categorized as being narrow or broad band. Narrow-band disturbances are further broken down into synchronous and nonsynchronous disturbances. Synchronous disturbances are due to noncircular tracks (written-in runout) and the center of the tracks not being coincident with the spindle rotation axis. Synchronous disturbances appear as sharp peaks at harmonics of the spindle rotation frequency in the disturbance spectrum.

The air currents (so-called "windage") that arise from the pumping action of the rapidly rotating disks give rise to both narrow-band and broad-band disturbances. These air currents cause nonsynchronous narrow-band disturbances by exciting disk flutter vibrations and mechanical resonances in the actuator assembly. Turbulent flow results in broad-band disturbances. However, the adoption of fluid-bearing spindles has eliminated the disturbances from ball and race imperfections and greatly reduced nonrepeatable runout from the spindle motor. Broad-band disturbances require high bandwidth to attenuate their effect, whereas narrow-band disturbances are amenable to localized high gain.

Noise and distortion are two other important sources of tracking error in disk drives. Noise arises not only from the electronics, but also from imperfectly aligned grains in the medium, at transitions and at the sides of tracks. The GMR head readers are nonlinear devices that introduce distortion and noise from domain-wall shifting. Quantization noise is 


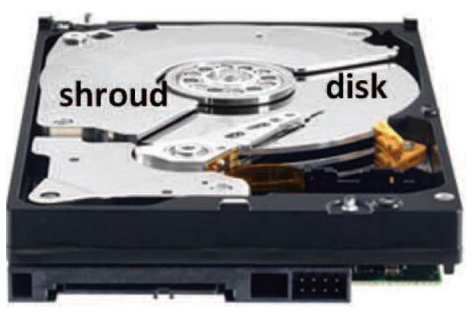

Fig. 10. Disk drive with a shroud for reducing windage disturbances on the actuator, at the cost of increased power consumption by the spindle motor. Squeeze film damping provided by the shroud can also reduce disk flutter.

of course present in any digital control system due to the analog-to-digital and digital-to-analog conversion processes.

\section{Improving Tracking Performance}

To improve tracking accuracy, there are three complementary goals of disk-drive research: 1) to decrease the disturbances themselves; 2) to increase the bandwidth of the track-following servo system; and 3) to better use the existing bandwidth to target specific disturbances. By itself, the servo would be insufficient to achieve the high track densities of today's commercial disk drives. The performance of HDD servo systems has benefited from numerous advances to reduce the magntitude or effect of disturbances themselves. The use of smaller components has increased the ratios of stiffness to inertia, thus increasing resonance frequencies and lowering the effects of external shock and vibration and of aerodynamic drag. Various shrouding mechanisms have been employed to reduce the air flow impinging on the actuator arm and suspension, see Fig. 10. Squeeze film damping provided by these shrouds can also reduce disk flutter. However, they also increase the drag on the disks, resulting in greater power consumption by the spindle motor. Slower spindle speeds in applications where the highest densities are needed, e.g., laptops, have also helped.

In general, the effect of disturbances on the positioning accuracy scales with frequency such that doubling the servo bandwidth reduces the PES magnitude by a factor of four at low frequencies but only a factor of two or less near the bandwidth frequency. Reductions in the size of components and clever design of the actuator arm and suspension have led to actuator bandwidths (open-loop $0 \mathrm{~dB}$ crossover frequencies) of nearly $2 \mathrm{kHz}$.

Nevertheless, research on design and control of dual-stage actuators to achieve higher bandwidth has continued at a brisk pace since IBM developed an electrostatic MEMS actuator in the mid 1990s [3]. The two most popular configurations are piezoelectric actuators mounted on a modified suspension and electrostatic actuators for moving the slider relative to the suspension [4], [70], [84]-[89]. One of the advantages of such dual-stage systems is that for short seeks, the emitted vibrations are significantly reduced owing to the very small inertia of the second-stage actuator compared with that of the VCM and arm. Another potential advantage is much faster seeks of just a few tracks, depending on the stroke of the actuator and the track density. Dual-stage actuators have appeared in some drives, see Fig. 11. Until recently, their presence in commercial products was still the exception rather than the rule because of cost and

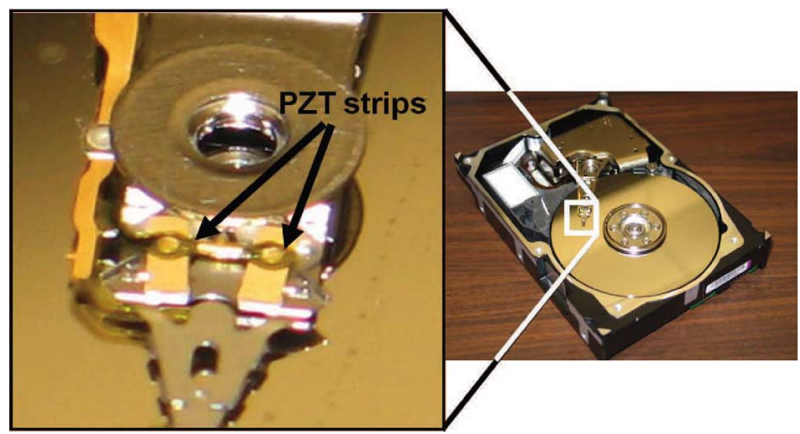

Fig. 11. Photo of a suspension-mounted piezoelectric milliactuator using leadzirconium-titanate.

the low stiffness they exhibit. They are becoming more common in enterprise level drives, as found for example in server farms.

Other hardware modifications for the servo have been proposed such as instrumented suspensions for active damping of vibrations [90], [91], and accelerometers for feedforward compensation of shock and vibration [92]. So far it appears that performance improvements offered by instrumented suspensions have not justified their cost. Accelerometers are used routinely on high-end drives for feedforward control. For low-end drives, they serve primarily as shock detectors for the disk drive to stop writing and to park the head to prevent damage.

Repetitive control is an excellent example of exploiting existing bandwidth more effectively to reduce TMR [93], [94]. Broadly broken down into feedforward and feedback algorithms, repetitive control algorithms provide compensating signals at harmonics of the spindle rotation frequency to address repeatable runout. Ultimately, the algorithms are examples of the internal model principle [95] because there must be some feedback to adapt the feedforward signal [96], [97]. Algorithms that provide high gain at every harmonic of the rotation frequency up to the Nyquist frequency have largely been replaced by phase-stabilized high-gain resonant filters that address just a few of the disturbances [98]. Although compensation of repeatable runout is a well-mined area, new results continue to emerge [99].

Robust control is another example of using existing bandwidth more effectively, particularly for compensation of broadband disturbances. $H_{\infty}, H_{2}$, and $\mu$-synthesis techniques have been applied extensively in recent years [88], [100]-[105]. The advantage of these powerful automated design techniques is that they provide the capability of guaranteeing performance in the presence of modeling uncertainty and the ability to balance disturbance rejection, noise attenuation, control effort, and potentially other aspects of system performance by the construction of a generalized plant with weighting functions. Their disadvantages are that they require a realizable state-space model of the plant and that the weighting functions must be the magnitude response of realizable state-space models. Also, the controller will have the same order as the generalized plant, and model reduction must often be employed. Thus, the design problem becomes one of choosing a good plant model and designing the weighting functions. While the software generating the controller runs quickly on today's computers, the selection of the weighting functions can be time consuming, and, if done poorly, can result in an overly conservative design. To some extent these 
disadvantages have limited the use of robust control methods for commercial drives. However, with the increasing prevalence of dual-stage systems, their utility for the design of controllers for MIMO plants is leading to their wide application.

An alternative to the design of a robust controller by considering a whole family of plants is a controller tuned to optimize the performance of each actuator on an individual basis. The concept of online tuning to optimize the performance of each actuator is investigated in [106].

A relatively recent development with a very significant impact on servo-system performance has been the application of an elegant but basic aspect of classical control theory. Phase stabilization exploits the fact that the magnitude response of a SISO system can cross $0 \mathrm{~dB}$ multiple times, provided the phase is such that encirclements of $-1+0 j$ on the Nyquist plot are avoided [107]. Fig. 12 illustrates the concept. By altering the phase response at a resonance, the energy of a vibration mode can be turned to advantage to reduce the effect of disturbances around that frequency, even if that frequency is above the 0 $\mathrm{dB}$ crossover frequency of the rigid body mode. This results in a prominent notch in the sensitivity function at exactly those frequencies that are most vulnerable to disturbances. The simplicity and effectiveness of this approach have led to its rapid adoption throughout the disk-drive industry. This phase-stabilization approach also motivated the development of several new controller structures or new uses for existing structures as well as new design techniques [98], [108], [109], and it has been extended to dual-stage actuator systems [110].

\section{Summary and Outlook}

To paraphrase the famous words of Mark Twain, the imminent death of hard-disk drives has been greatly exaggerated. The total storage capacity of all shipped disk drives greatly exceeds the conceivable storage from solid-state memory for the foreseeable future. The servo systems of disk drives continue to improve thanks to better components and good engineering to reduce disturbances. Dual-stage actuators are found in some products, primarily enterprise drives, even though their costs and limitations have so far prevented their widespread use. Sophisticated control design approaches and architectures have been employed for seeks and track following [111] and [112], whereas the simple approach of phase stabilization has greatly improved the performance of servo systems. Newer work on preview control may significantly improve seek performance, particularly for dual-stage systems.

In the future, servo engineers may be required to design controllers for fly-height control. Currently thermal actuators are used in some drives to lower the W/R head toward the disk surface, which relaxes the fly-height requirements. The control of this actuator is open-loop after calibration. As data-storage densities increase, it may be necessary to lower the head even further, which will require feedback control to avoid collision between head and disk.

Candidates for achieving densities higher than $1 \mathrm{~Tb} / \mathrm{in}^{2}$ include heat-assisted magnetic recording (HAMR), use of patterned media, a combination thereof, or something entirely different [113]. The servo will be affected by the choice of technology. For example, skew compensation may be necessary to align the $\mathrm{W} / \mathrm{R}$ head for patterned media, or to align the heating

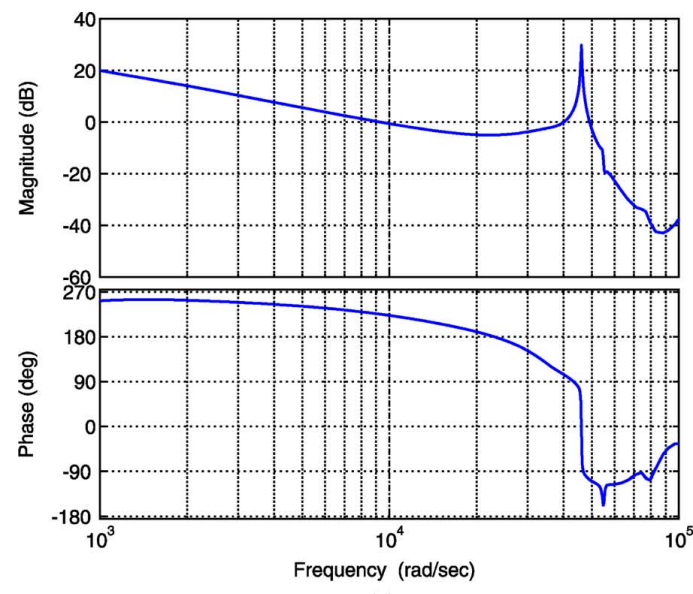

(a)

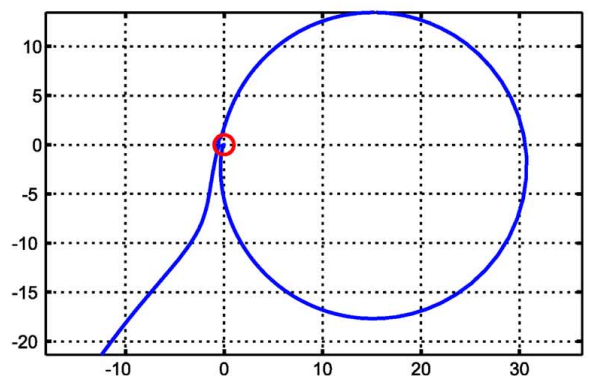

(b)

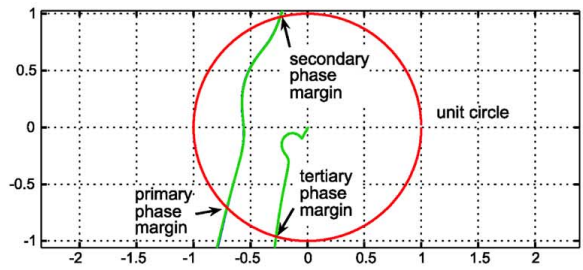

(c)

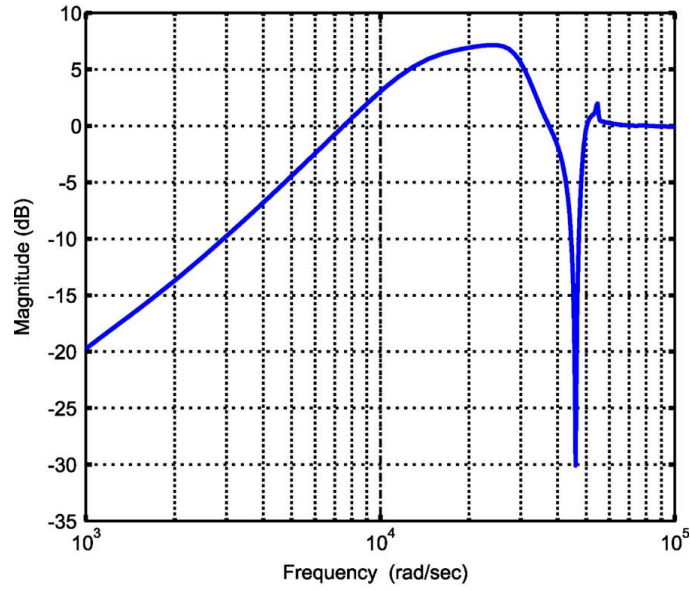

(d)

Fig. 12. Illustration of the phase-stabilization method. (a) A SISO system with multiple $0 \mathrm{~dB}$ crossovers. (b) and (c) Corresponding Nyquist plot. The system will be stable in closed loop if the Nyquist plot does not encircle the point $-1+0 j$, even though the open-loop frequency response crosses $0 \mathrm{~dB}$ past the $-180^{\circ}$ frequency. (d) The sensitivity function will feature a prominent notch at the frequency of the open-loop peak. The portions of the Nyquist plot in (b) and (c) outside of the unit circle (red) correspond to those portions of the Bode plot (a) with magnitude greater than $0 \mathrm{~dB}$. The nearly complete circular arc corresponds to the resonance at approximately $4600 \mathrm{rad} / \mathrm{s}$.

element and magnetic element of a HAMR head. Also, PES generation may become very different. The servo engineer's job is 
far from being over, and it will continue to be an interesting endeavor in the years to come.

\section{Optical Data-Storage Systems}

The optical data-storage industry has been in existence for almost three decades, as compact disks (CDs) were first introduced in the consumer market in 1982 [114], [115]. Initially, optical storage technology was thought to dominate the future storage markets because of its higher data density than available in the incumbent magnetic tape and HDD technologies [116]. Magnetic technologies, however, have evolved faster than optical technologies. The relatively lower capacity and data transfer rate of optical data-storage systems, usually referred to as ODDs, explain their market share as a portable interchange medium between computers instead of being used as a primary data-storage system. Moreover, their low cost and easy reproducibility made them good candidates for information distribution and archiving applications. Although portable storage, especially for mobile devices, now has been taken over by Flash memory, optical products are expected to continue to complement and coexist with HDDs because of their unique advantage of being a low-cost removable medium [116]. To date, ODDs, such as CD-ROMs and DVDs, are the most widely used data-storage systems. Recently, Blu-ray disks began to become popular devices because of their higher data-storage capacity. Servo-control technology has been a key aspect in ODDs poised to evolve towards higher data capacity because of its important role of moving the optical pickup to a track and controlling it to read/write data along the track.

\section{A. General Architecture}

1) System Architecture: A typical example mechanical system with an optical pickup unit (OPU) is shown in Fig. 13. The OPU is mounted on a sled (coarse actuator) controlled by either a stepping or a direct-current (DC) sled motor. The medium is mounted on the spindle system, with the spindle motor running either in constant linear velocity (CLV) or constant angular velocity (CAV) mode. The generic ODD architecture and servo system are well described in [114], [115], [117], and [118]. An OPU includes several components: 1) servo components: focus actuator, (fine) track actuator, and springs that hold the objective lens; 2) optical components: laser diode, collimation lens, polarizing beam splitter, objective lens, etc.; 3) electric components: monitoring photo-detector (MPD), photo-detector IC for channel signals, etc. To read (record) data from (to) an optical disk, the so-called servo system should control the OPU, the sled motor and the spindle motor in the correct sequence. Details on the functionality of the control loop in commercial ODDs can be found in [114], [115], [119], and [120].

2) Servo Actuators in $O P U$ : The control quality of the focus and track servo systems mainly determines the performance of an ODD. Hence the design parameters of an OPU can be a major concern. As illustrated in Fig. 13, a laser beam traverses the collimation lens, polarizing beam splitter, quarterwave plate, and objective lens before reaching the medium. The light reflected from the medium then passes through the objective lens, quarter-wave plate, and detection lens before

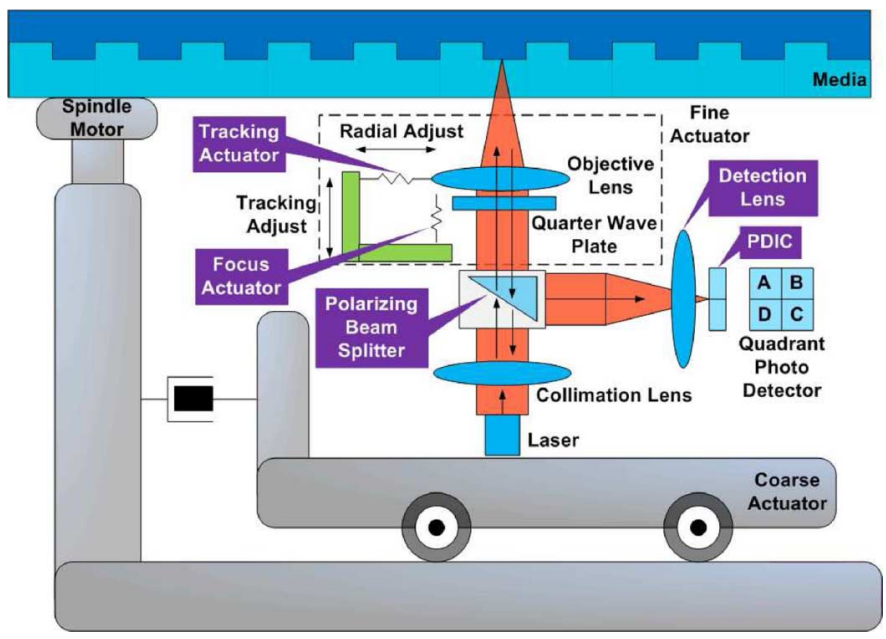

Fig. 13. Optical pickup unit [115], [118], [121].

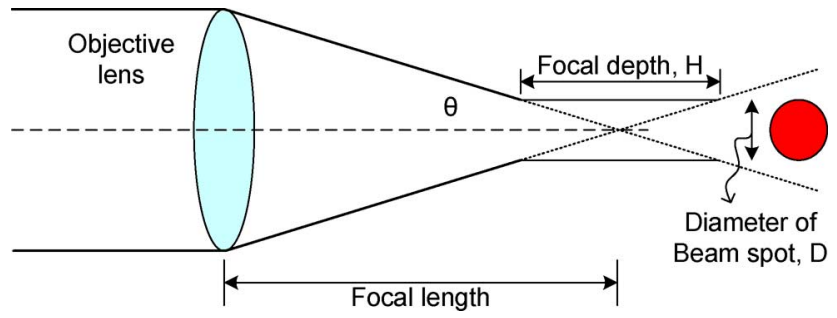

Fig. 14. Principle of focusing.

reaching the photo detectors. To provide side beams, a diffraction grating can be placed between the laser and collimation lens. Side beams follow the same path as the main beam, but are detected on side-beam detectors half a track away. They are focused on the medium as shown in Fig. 14. Except for the media, all components are placed on the optical pickup on top of the coarse actuator [115], [118]. In general, the servo actuators can be modeled by a mass-spring-damper system [120], [122], [123], and the actuation to an objective lens for focusing (or tracking) is achieved by a voice-coil actuator. Thus, given the electrical components of the actuator with resistance $R$ and inductance $L$, the overall transfer function from the input voltage $V(\cdot)$ to the displacement $X(\cdot)$ can be described by [115]

$$
\frac{X(s)}{V(s)}=\frac{1}{R+L s} \cdot \frac{K}{\left(\frac{s}{\omega_{n}}\right)^{2}+\frac{1}{Q}\left(\frac{s}{\omega_{n}}\right)+1}
$$

where $\omega_{n}$ is the natural frequency, $Q$ denotes the $Q$-factor, and $K$ is the DC gain from the current to the displacement. The electrical dynamics is often neglected and modeled as a secondorder system by using a current amplifier with proportional and integral (PI) feedback control [120].

3) Focus-Error Detection: The focus error (FE) is determined by using an astigmatic objective lens and a sensor for the reflected light, which contains four separate photodiodes that divide the sensing area into separate quadrants as shown in Fig. 13. The basic principle for creating a focused beam spot on the reflective layer on a disk is shown in Fig. 14. Once an objective lens is located at a focal length from the reflective layer, the beam spot can be generated within the focal depth range with a 
TABLE I

COMPARISON OF OPTICAL PARAMETERS

\begin{tabular}{|c||c|c|c|}
\hline Parameters & CD & DVD & Blu-Ray (BD) \\
\hline Capacity/layer $(\mathrm{GB})$ & 0.7 & 4.7 & 25 \\
\hline Layers/disk & 1 & $1,2,4$ & 1,2 \\
\hline Track pitch $(\mu \mathrm{m})$ & 1.6 & 0.74 & 0.32 \\
\hline$\lambda(\mathrm{nm})$ & 780 & 650 & 405 \\
\hline $\mathrm{NA}$ & $0.45-0.5$ & $0.6-0.65$ & 0.85 \\
\hline$D(\mu \mathrm{m})$ & $1.56-1.73$ & $1.00-1.08$ & 0.476 \\
\hline$H(\mu \mathrm{m})$ & $3.12-3.85$ & $1.54-1.81$ & 0.561 \\
\hline Speed $(\mathrm{Mb} / \mathrm{s}$ at $1 \times))$ & 1.2 & 11 & 36 \\
\hline Operating speed $(\times)$ & $1-52$ & $1-16$ & $1-16$ \\
\hline
\end{tabular}

Focus error
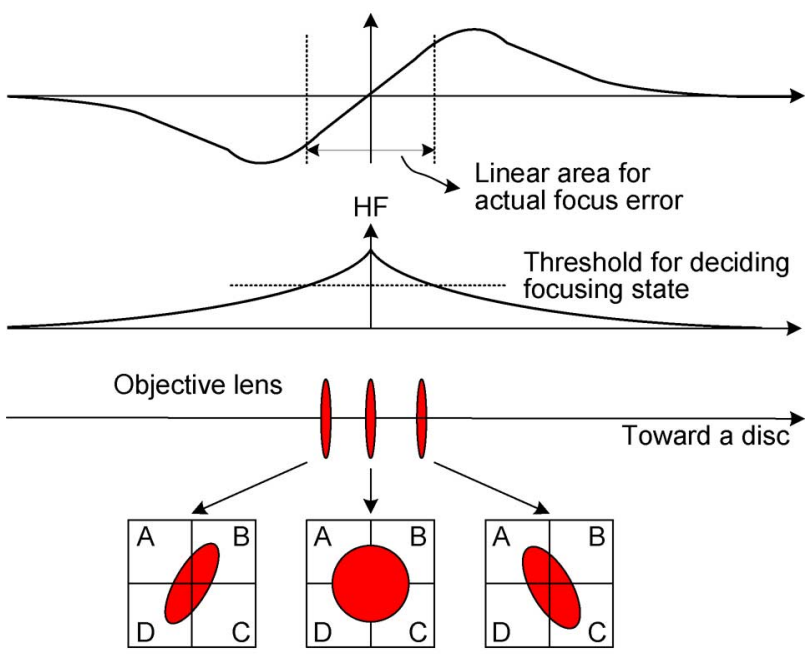

Beam spots in 4 quadrants of a photo-detector

Fig. 15. Focus-error detection based on an astigmatic method.

certain diameter because of the spherical aberration of the objective lens. The numerical aperture (NA) of an objective lens is defined by $N A=n \sin \theta$, where $n$ is the refraction index. Then, observe that

$$
D=\frac{\lambda}{N A}, \quad H=\frac{\lambda}{N A^{2}}
$$

where $\lambda$ is the laser wavelength, and $D$ and $H$ are the diameter of the beam spot and the focal depth, respectively. The optical parameters of the media are summarized in Table I. We refer to [8] for further details.

Several methods for FE detection exist [114], [115], [118], [124], [125]. Fig. 15 shows a typical example of generating the $\mathrm{FE}$ and high-frequency (HF) signal using the sub-signals of a main photo detector. The shape of the beam spot on the quadrant photo detector depends on the distance between the disk and the objective lens. The HF signal is defined by

$$
H F=A+B+C+D
$$

where $A, B, C$, and $D$ are the signal levels of the channels. When the objective lens is located exactly at its focal length from the disk, the beam spot is evenly distributed on the four channels and HF achieves a maximum. From Fig. 15, we see that the FE is approximately linear within a certain range. Then a FE that is above a certain level is defined as

$$
F E=A+C-(B+D) \text {. }
$$

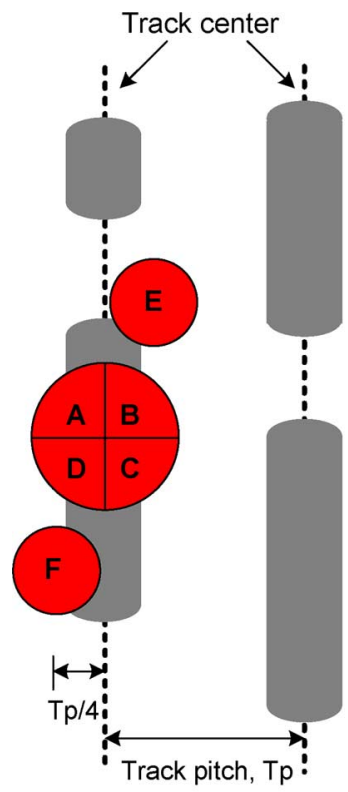

(a)

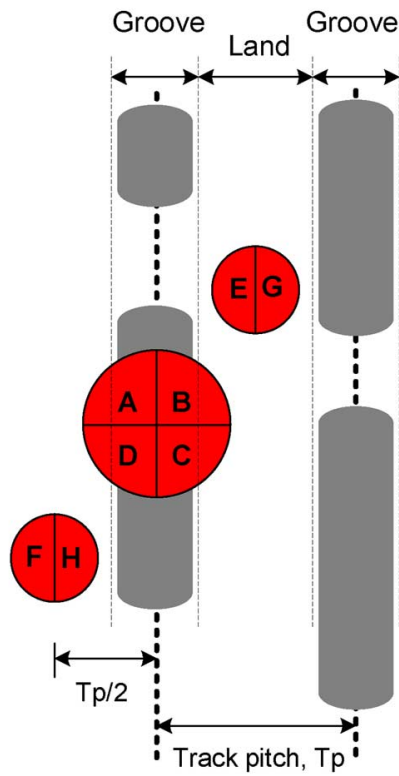

(b)
Fig. 16. TE detection by (a) three-beam and (b) differential push-pull method. The data pits are at the center of the groove, and the side detectors help provide more accurate TE signals.

As the objective lens moves up and down towards the medium, see Fig. 15, the FE is expected to assume an $S$-curve behavior around the focal length defined in Fig. 14.

4) Track-Error (TE) Detection: The TE measures the distance of the laser spot from the desired track. Several methodologies for TE detection exist for disk media [118], [126], [127]. In this paper, we discuss three main methods used in practice, namely, the three-beam method, the differential push-pull method, and the differential phase-detection method. The three-beam method was developed for CDs with data pits, such as CD-ROMs. In a CD OPU, the laser is split into three beams by a refraction-grate plate as shown in Fig. 16(a). At the same time, two other sub-beams produce $E$ - and $F$-channels in a photodetector. The TE can be defined as

$$
T E=E-F .
$$

The differential push-pull (DPP) method was devised for CD media with land-groove architecture, such as CD-R or CD-RW disks. As shown in Fig. 16(b), the sub-beams are located at a distance of one half the track pitch. The height gap between land and groove and the inclination of the area connecting the two result in a difference that is correlated to the track deviation in the main beam push-pull (PP) signal

$$
P P=A+D-(B+C) .
$$

This PP signal is very sensitive to the disk tilt so that four additional channel signals obtained from two sub-beams must be used to resolve this matter. The side-beam push-pull (SPP) signal

$$
\mathrm{SPP}=E+F-(G+H)
$$


may contain an offset component, which renders also the mainbeam PP signal sensitive. Thus, one can define a differential PP signal as follows:

$$
\mathrm{TE}=\mathrm{PP}-\mathrm{SPP} .
$$

The differential PP method is robust against disk tilt and good for CD-R or CD-RW media.

Differential phase detection (DPD) is a TE-detection method devised for DVD playback. It can be extended for DVD-Recordable (or rewritable) media with land-groove structure, but DVD-ROM disks (without land-groove structure) need a different TE-generation method. To resolve this, DPD uses four main channel signals for detecting the phase difference [118], [127]-[130].

Phase-shift DPP (PS-DPP) is used for precise alignment of the three spots on the track edge for the different track pitches of multi-type DVDs [131].

5) Servo Loops: There are six major control loops in ODDs: fine tracking, coarse tracking, focus, spindle, phase-lock loop (PLL), and laser power [118]. The typical architecture of an ODD and the general design of a servo system for reading data from a disk are summarized in [130]. For CD drives, these functions are well described in [115]. Good descriptions of each loop can be found in [118]. The servos of the fine-tracking and the focus loop typically include a lag-lead or proportional-integral derivative (PID) compensator with notch filters, or a state-feedback controller with an estimator [115], [120]. The objective lens is generally held by a set of springs as shown in Fig. 13. The plants may be modeled as simple mass-spring-damper systems with two states, namely, position and velocity, in a state-space model. A bias force can be included in the model as an augmented state for tracking to represent the friction force from the rails on which the coarse actuator slides and the tension force from the connecting cable.

The combined fine and coarse-tracking loop is illustrated in Fig. 17. Although fine tracking (objective-lens movement) and coarse tracking (sled movement) are physically coupled, they can be considered as being decoupled because of the difference in their frequency range of operation: the sled loop has a much lower bandwidth than the fine-tracking loop. A master-slave control method can be employed such that the fine-tracking loop uses the TE signal filtered by a high-pass filter, whereas the coarse-tracking loop uses a low-pass filter. The lower-bandwidth loop commands the sled to position the OPU, whereas the higher-bandwidth loop drives a VCM actuator for fine positioning of the objective lens. The amount of repeatable runout (RRO) is estimated and injected as a feedforward term as is done with the bias force. The focus loop depicted in Fig. 18 is similar to the fine-tracking loop because the lens dynamics in the radial and the vertical direction are similar. As the TE is of sinusoidal shape, zero crossings can be used to count the number of half tracks during a seek. Details on the spindle, PLL, and laser control can be found in [114], [115], and [118].

\section{B. Control Issues and Methods}

1) Focus Servo: The focus servo is the first of several feedback loops that are active in an ODD. Hence, achieving reli-

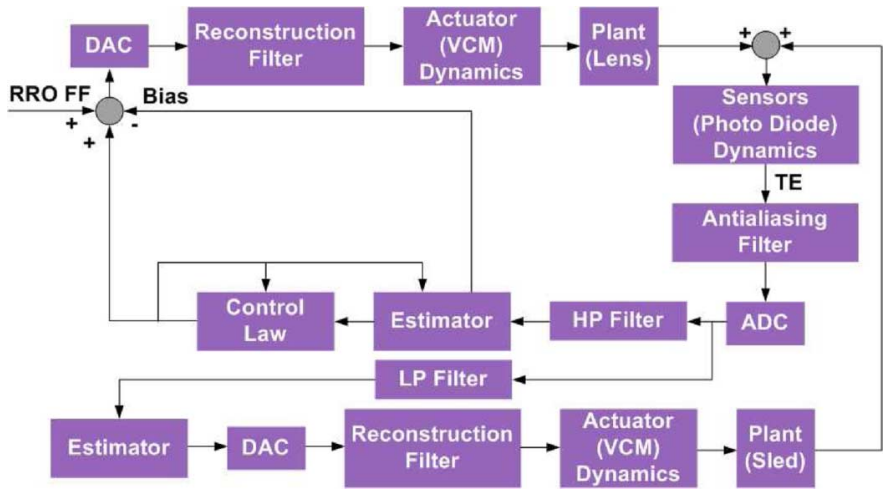

Fig. 17. Tracking control loops [118].

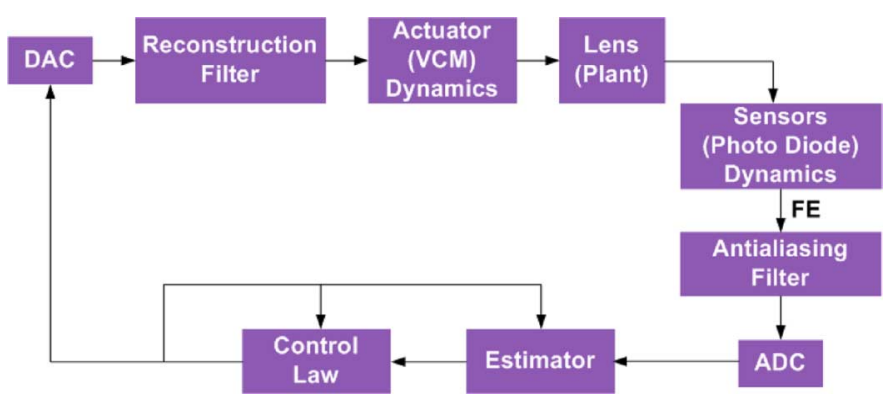

Fig. 18. Focusing control loop [118].

able focus control is a key factor for the performance of data reading (or recording) [132]-[135]. In general, the vertical deviation of a disk should be less than $\pm 1 \mathrm{~mm}$, which implies that the pickup should be designed to allow a working distance of \pm 1 $\mathrm{mm}$. The open-loop gain of the focus servo in the low-frequency band covering the rotational frequencies should be increased appropriately to suppress the effect of the vertical deviation. As severe vibrational disturbance weakens the performance of the focus control [134], the bandwidth and gain of the focus servo should be determined for the specific targets envisioned. Standardized vertical deviations of the information layer specified at the disk-scanning velocity of CD-ROM drive can be found in [115].

2) Track Servo: The track servo is needed for reading the data from a spiral track [118], [122]. To accomplish the trackfollowing operation in an ODD, both the pull-in and the steady track-following task must be performed reliably [136], [137]. The eccentricity of a track is defined by the mismatch of the rotational center with respect to the geometrical center of data tracks. The main sources of the eccentricity are inaccurately formed tracks on the medium and poor clamping of a disk. In practice, for CD-ROMs, an eccentricity of $210 \mu \mathrm{m}$ is the maximally allowable eccentricity a track servo can cope with. When a disk exhibits a certain amount of eccentricity as it rotates, the beam spot (emitted from the objective lens) follows a relative trajectory on the disk that results in a sinusoidal track error when the beam spot crosses the data tracks. To follow a data track, the track servo or track controller should be turned on when data track is crossed so that the track controller can suppress the radial velocity and stably pull in the beam spot onto the data track. After the pull-in phase, the track servo should 
sustain the track-following operation. Eccentricity also affects the track-following performance, in particular at high rotational speeds. Note that the tracking servo in an ODD is formulated by an unknown reference tracking problem, and the unknown reference fluctuates with the amount of eccentricity, synchronized with the rotational frequency. Thus, the unknown reference can be treated as an unknown disturbance, which can be handled by increasing the low-frequency gain of a track controller.

The allowable amount of track position error is defined by the device specification (see [115] for CDs). Boosting the low-frequency gain of the controller may weaken the phase-lead property around the cutoff frequency and thus degrade the dampening property. To avoid this difficulty in designing a nominal track servo, various approaches have been proposed based on disturbance observers [136], [138]-[141], repetitive control [121], [142]-[144], or nonlinear control [145].

In addition to eccentricity and black dots or scratches on a disk, the robustness of the track (or focus) servo is one of the major issues affecting performance.

3) Robust Control: ODDs are affected by various disturbances related to disk imbalance, eccentricity, windage, external shock, and resonances caused by the actuator itself. These disturbances contain significant periodic components appearing at a known fundamental frequency, which corresponds to the disk rotational velocity, and higher harmonics. Disturbance observers (DOBs), repetitive control, and iterative learning control (ILC) have been successfully applied for rejecting periodic disturbances or disturbances at low frequency ranges.

DOBs have been applied to enhance the tracking performance [146]-[149]. Because in ODDs only the TE signal is available, a modified estimation method achieves improved performance [150], and multi-loop DOBs provide further improvement [151]. Robust DOBs that take the model uncertainty into account have also been used [152], [153]. By considering the pull-in capability, steady track following can be achieved by an add-on DOB designed in the time domain [137], [139]. At high rotational frequencies, modifications to conventional DOBs achieve better performance than conventional DOBs [154], [155].

The advantages and disadvantages of repetitive control are well summarized in [96] based on four different algorithms to cancel periodic disturbance. A tutorial on iterative learning control and repetitive control is given in [156]. The drawback of repetitive control is that exact knowledge of the period of the external signals is required. In practical applications, the period must be constant or else an accurate measurement of the periodicity is necessary. Although the method described in [157] requires additional memory elements, it is very effective for rejecting disturbances when the period varies within a narrow range.

Another drawback is due to the Bode sensitivity integral: perfect reduction at the harmonic frequencies is counteracted by amplification of noise at intermediate frequencies. Various approaches have been reported to address this problem (for details, see [142]). In [121], for example, a sufficient condition for robust stability of the repetitive control system is derived in the form of a linear matrix inequality (LMI). In [158], a modified repetitive compensator uses notch filters with feedforward gain.

The goal of ILC is to generate a feedforward control that tracks a specific reference or rejects a periodic disturbance. Recently a survey of ILC has been published [159] that looks at the analysis, design, time, and frequency-domain system representation. The convergence conditions of learning control schemes have been explored in detail in [160].

An add-on-type output regulator is able to reject sinusoidal disturbance at a known frequency [141]. This approach has been extended to an adaptive output regulator to reject the sinusoidal disturbances of unknown bias, magnitude, phase, and frequency [161] using a modified adaptive algorithm [162]. Novel frequency adaptive control techniques (FACTs) to deal with the RRO cancellation for both CAV and CLV spindle modes in ODDs were studied in [163] and [164].

There are also nonperiodic disturbances, such as surface defects and external shocks. Surface defects, for example, are scratches, fingerprints or dust on the disk surface. They distort tracking error signals reflected by the disk surface, and consequently deteriorate the track-following performance. The robust controllers designed to reject RRO are unable to effectively eliminate nonperiodic disturbances [165], [166].

A summary of fault detection and shock disturbance compensation methods is presented in [130]. A comprehensive study on surface defects can be found in [167] and [168]. The use of estimators to obtain accurate TE information when the optical pickup passes surface defects has been considered in [135], [169], and [170]. A nonlinear dynamic filter providing an additional gain to the feedback controller can be used to improve disturbance rejection [145], [171]. An experimental performance analysis based on frequency-domain measurements has also been made [172]. Switching control using an observer was shown to be effective in track following even in the presence of disk scratches or heavy fingerprints [173]. A simple additional current measurement that provides positive feedback effectively eliminates disturbance and thus attenuates high-frequency disturbances such as mechanical shocks [174]. A robust servo-control system using observer-based sliding-mode control is presented in [175]. Although these systems enhance the anti-shock performance, the control structures are complex. In a recent study, a nonlinear anti-shock controller having a dead zone improved the stability margin without degrading tracking performance [10].

\section{Near-Field Recording Systems}

The data density of an optical recording medium depends on the size of the focused laser beam spot. This can be reduced by using a shorter-wavelength laser or a larger NA objective lens (see Table I). Several new far-field optics-based approaches, e.g., the magnetic super-resolution (MSR) and the optical super-resolution (OSR) technology, have been proposed to further increase the recording density, but cannot achieve more than 100 GB capacity [9], [176].

Optical far-field-based approaches have reached the theoretical and technological limits because of the diffraction limit. A focused laser beam can read out recorded pits with the real 


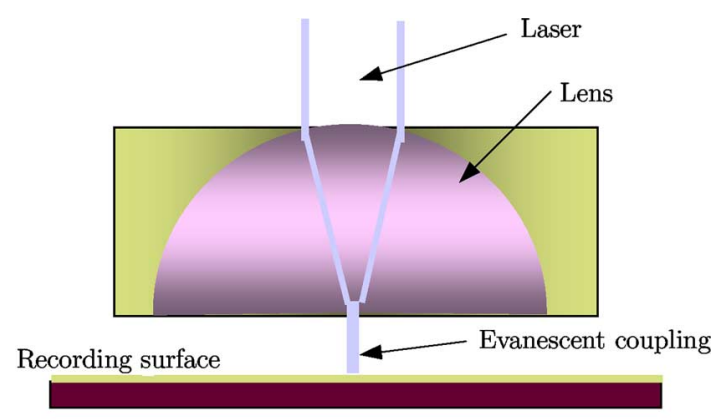

(a)

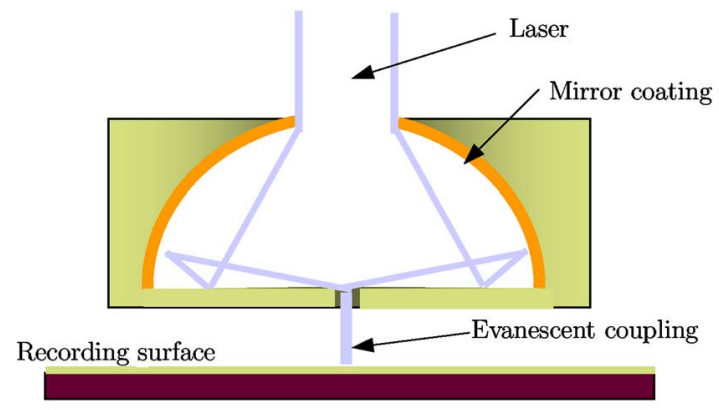

(b)

Fig. 19. Optical near-field recording: (a) SIL and (b) SIM.

cutoff frequency determined by the rate of the spatial cutoff frequency $(2 \mathrm{NA} / \lambda)$ times the disk rotation speed. The diffraction limit, therefore, determines the data-storage capacity of a disk. To overcome this resolution limit, the use of optical near-fields, which provide a significantly smaller beam spot, has been suggested [9], [176]-[179]. The optical near-field is a special electromagnetic field that can achieve a significantly smaller laser beam spot size to overcome the resolution limit, but cannot propagate over long distances (unlike optical far-field waves).

Optical near-field recording (NFR) refers to the extremely short distance (called air gap, approximately $25 \mathrm{~nm}$ ) between the objective lens and the disk surface. The gap is much shorter than that of CDs and DVDs (roughly $\sim 1 \mathrm{~mm}$ ) or even of BDs $(1 \mathrm{~mm})$. This means that much smaller marks $(<100 \mathrm{~nm})$ can be recorded and read out by different optical probes to achieve more than $100 \mathrm{~GB}$ capacity in a $12-\mathrm{cm}$ disk. The optical nearfield, however, cannot propagate beyond $100 \mathrm{~nm}$, and its intensity decreases exponentially with the distance. Accordingly, the medium surface has to be ultimately flat compared with current removable media, such as DVDs and CDs, and control of the flying height, i.e., the air gap, becomes critical.

The development of a high-NA lens system with a low-flying recording head is very challenging. A promising approach is to use a blue laser to write and read data through a solid immersion lens (SIL) or solid immersion mirror (SIM), both of which significantly reduce the spot size of the focused beam at the bottom of the lens as illustrated in Fig. 19 [9], [178]-[180]. Other optical NFR methods proposed have practical limitations. A thin-film technique called "super-RENS" [181] has a very low SNR. Here a gap servo using a PZT actuator was introduced [182], which requires a high control voltage and a short working distance with hysteresis.
Although the system structure of NFR appears to be very similar to that of conventional ODDs, the air-gap width is much smaller. In optical NFR, the positioning control accuracy required is far below $100 \mathrm{~nm}$, which may not be attainable using current optical far-field device control technology. Mode switching, i.e., open-loop mode for approach and closed-loop mode for air-gap control, is widely used in air-gap servo control, as the gap error signal (GES) is available only in the near-field distance because of its non-flat shape and clamping. The (open-loop) approach mode brings the lens head from the far-field into the near-field distance, and then closer to the surface. When the lens enters the near-field range, the air-gap controller is switched to closed-loop mode. Sony demonstrated $20 \pm 1 \mathrm{~nm}$ at a $150-\mathrm{rpm}$ disk $(2.5 \mathrm{~Hz})$ [183], whereas Philips recently demonstrated $20 \pm 1 \mathrm{~nm}$ air gap at a $3500 \mathrm{rpm}$ (58.3 $\mathrm{Hz}$ ) polycarbonate disk [184].

For track seek and track following in NFR, conventional methods can be used, although a more challenging servo-control specification is required. During track-seek servo, the head should be set as far away from the disk as possible (the maximum working distance of the biaxial device) to avoid collisions between the SIL head and the disk. The gap servo system provides an open-loop control signal to the head actuator to make the head approach the disk [184]-[187], possibly along a specific profile to reduce the pull-in time and avoid overshoot that may cause collisions. Because of the delay of the air-gap error signal, the introduction of an appropriate open-loop control signal is desirable to reduce the pull-in time and to prevent overshoot [188]. Use of a DOB to reduce the overshoot at the switching point between open-loop and closed-loop modes was demonstrated in [189].

\section{Holographic Data Storage (HDS)}

HDS using holographic technology, which has been researched since the 1960s for its data-storage potential, is capable of storing more than $100 \mathrm{~Gb} / \mathrm{cm}^{3}$ and transferring data at speeds faster than $1 \mathrm{~Gb} / \mathrm{s}$. Thus HDS has been considered suitable for archival systems and massive multimedia data storage. For details on HDS systems, the reader is referred to [11], [190], [191], and the references therein. In [192], fundamental issues underlying HDS, such as grating formation, recording and readout of thick and thin holograms, multiplexing techniques, SNR considerations, and readout techniques suitable for conventional, phase-conjugate, and associative search data retrieval, including a discussion on electronic implementations for control, signal encoding, and recovery, are extensively reviewed. A roadmap for optical data-storage applications, including a comparison of the storage density of HDDs to that of optical data storage including HDS, is presented in [116]. A tutorial on servo techniques for holographic storage system can be found in [193]. Details on servo and drive control for system implementation are presented in [194]. HDS systems store data in the form of holograms, which are images of 3-D interference patterns created by the intersection of two beams of light in a photosensitive storage medium. In general, both page-based and bit-wise holographic techniques have been pursued. For various approaches to HDS, the reader is referred to [192]. 
In page-based HDS, a signal beam containing digitally encoded data is superposed on a reference beam within the volume of the storage medium, resulting in a chemical reaction that changes or modulates the refractive index of the medium in the volume. This modulation serves to record both the intensity and the phase information of the signal. Each bit is therefore generally stored as a part of the interference pattern. The hologram can later be retrieved by exposing the storage medium to the reference beam alone, which interacts with the stored holographic data to generate a reconstructed signal beam proportional to the initial signal beam used to store the holographic image. The two primary approaches to increase capacity exploit a multiplexing approach via angle [193] and collinear phase conjugation [195]. Holoplex [196], Samsung [197], and Daewoo [198] also pursued angle-multiplexing methods. Optware [195] has been pursuing collinear phase conjugation [194]. InPhase developed an angle-polytopic phase conjugate using a monocular architecture for consumer applications [193], [194]. Coaxial-type holographic disk systems which use microreflector hologram have been researched by Samsung [199] and Sony [200].

In bit-wise holography or microholographic data storage, every bit is written as a microhologram or Bragg reflection grating, typically generated by two counter-propagating focused recording beams. The data is then retrieved by using a read beam to reflect off the microhologram to reconstruct the recording beam. Accordingly, microholographic data storage is more similar to current technologies than page-wise holographic storage. However, in contrast to the two layers of data storage that can be used in DVD and Blu-ray disk formats, holographic disks can have 50 or 100 layers of data storage, providing data-storage capacities that can be measured in terabytes. Moreover, as in page-based holographic data storage, each microhologram contains phase information from the signal. Detailed discussions on the two methods can be found in [201].

1) Servo Control: Many methods have been proposed to increase the capacity and data transfer rate using multiplexing and new storage materials. Angle, wavelength, and phase code methods have been introduced for multiplexing [190], [191], [194]. To use volume holography as a storage technology, the digital data to be stored must be imprinted onto the object beam for recording and then retrieved from the reconstructed object beam during readout. The input device for the system is a spatial light modulator (SLM) including a digital micromirror device (DMD). The SLM is a planar array of several thousand pixels. The output device is a similar array of detector pixels, such as a complementary metal-oxide-semiconductor (CMOS) sensor or charge-coupled device (CCD). To obtain reliable data, such as read-back data from HDS systems with a low bit-error rate (BER), it is necessary to control a reference beam precisely to align a holographic image on the image sensor. To achieve this objective, the reference beam should be positioned on the center line of the data track. Thus, it is important to have a tracking control method that is suitable for HDS systems. However, methods for designing and evaluating a tracking controller for an HDS servo system using disk media have rarely been reported. For disk-type media, a few methods for servo-signal generation have been proposed.

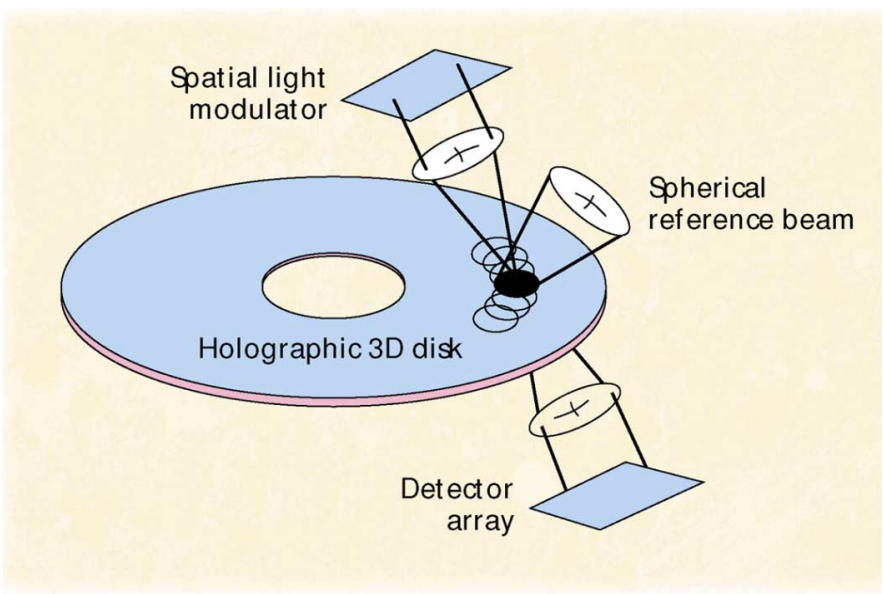

Fig. 20. Shift-multiplexed disk system: track position and disk rotation enable access to densely overlapping holograms [11].

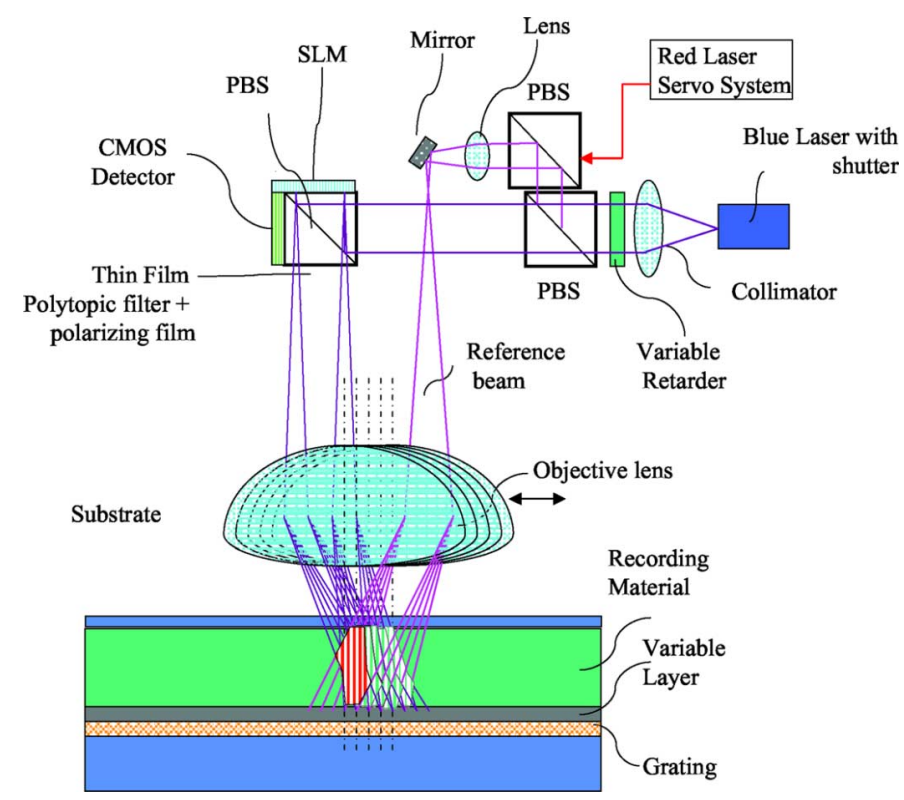

Fig. 21. Multiplexing angle-scan system configuration of HDS developed by InPhase [193].

Holoplex and InPhase use spatial multiplexing, which is the conventional two-axis holography as illustrated in Figs. 20 and 21. It is claimed that collinear technologies have significant advantages over conventional two-axis holography: good write and read performance, uniform shift selectivity for both radial and tangential directions, and fairly large system tolerance. Fig. 22 illustrates the optical configuration using collinear holography released by Optware. The introduction of Optware's mechanism enables reduced pickup size, elimination of vibration isolators, high-level compatibility with DVDs and CDs, and low-cost operation, effectively eliminating the remaining obstacles to full commercialization.

Optware uses a servo signal generated by prewritten pits, whereas Holoplex assigns a part of the holographic image for tracking error signal (TES) generation. Both methods use page-type data [196], [202]. Optware writes pits on a disk for generating a servo signal before writing data on the medium [195]. In this method, two separately controlled lasers are used 


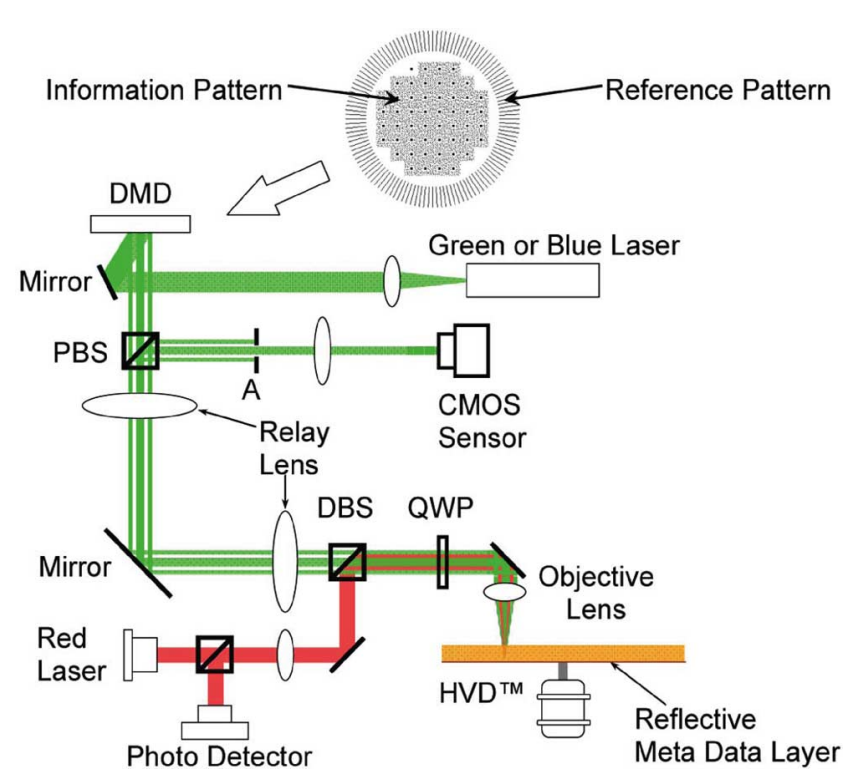

Fig. 22. Optical configuration of the HVD drive system using collinear technology [195].

for reading data, and the servo signal is obtained from detecting signals caused by laser reflections from the prewritten pits. Holoplex, in contrast, generates a servo signal using part of the holographic image. Its holographic image consists of a data image and a servo image. The data are read back through the image sensor, and the servo image is projected onto a photo detector (PD), or a part of the image sensor is used to produce the servo signal. InPhase uses a dedicated servo track with a sinusoidal encoder at the inner diameter (ID). Two servo systems based on the holographic signal recovered are designed to control the reference beam pitch angle and wavelength compensation requirement unique to page-based holographic memory system. Compensation for pitch angle misalignment and changes in the holographic medium dimensions are investigated in [194], where also the alignment principle for sequential recovery using wobble servo is introduced.

2) Tracking-Error Signal and System Tolerances: Optware uses two separately controlled lasers for reading data, and the servo signal is obtained by detecting signals caused by laser reflections from the prewritten pits. In the recording process, the information pattern in the center and the reference pattern in the outer ring are displayed simultaneously by the same SLM, and the information beam and the reference beam interfere with each other in the disk through a single objective lens. In the reconstruction process, only the reference pattern is displayed on the SLM. The reconstructed beam is sent back to the same lens by a reflective interlayer in the disk and reflected to a CMOS image sensor by a polarized beam splitter. A red laser is used for servo control and for locating holograms.

In contrast, Holoplex makes the servo signal using a part of the holographic image [196]. In Fig. 20, a shift-multiplexed disk system is depicted. It resembles a CD, with an SLM and detector array for parallel input/output, and a simple reference beam. Track position and disk rotation enable access to densely overlapped holograms [11].
In [197], it was shown that valid TES is available only intermittently, namely, when the level of the RF sum signal is high. To cope with this, a mode-switching control method was introduced. The tracking controller regulates the tracking error only when valid TES is generated, whereas a feedforward controller is used when valid TES is not available. A shift-compensation algorithm eases the tolerance position error bound. To understand the tolerances of HDS systems, a definition of SNR that relates to the BER is chosen [194]. SNR degradation arises from several causes, such as imperfect phase conjugation of the recovered data beam, Bragg mismatch, book-to-book crosstalk, and clipping by the polytopic filter. Further details on the tolerances are listed in [194].

3) Other Aspects: Using a pre-format layer facilitates tracking-servo control [203]. However, to avoid the use of a pre-format layer and an additional laser diode, using a holographic servo track to detect TES and perform tracking-servo control is possible [204]. In this method, the TES is available continuously, and standard techniques can be applied for tracking control. As the angle margin of the reference beam is very small (less than $0.01^{\circ}$ ), an angle servo is necessary [198]. When neighboring tracks are not recorded at the same diffraction efficiency and the average diffraction efficiency of one track is larger than that of adjacent tracks, this method causes an offset of the angle error signal [194]. For holographic systems capable of phase encryption a solution to ease the mechanical tolerances for positioning exists [205]. The phase modulation for the position marker image makes the readout image sensitive to the hologram positional error. The performance of some HDS systems using holographic disks may be sensitive to the tilt disturbance because of the rotational mechanism due to the Bragg effect. Therefore it may be necessary to detect and compensate the angle of the disk tilt [206]. It was reported that the tolerance of tilt angle (tangential tilt) is below $0.01^{\circ}$ for $3 \mathrm{~dB}$ loss of SNR when angle multiplexing is used. Basic tolerances of InPhase HDS systems were reported in [207]. Detecting the amount of tilting and its compensation with a conventional control method were successfully performed after filtering PES with a fourth-order low-pass filter to reject measurement noise, as reported in [208].

\section{Probe-Based Data Storage}

The emergence of a wide variety of mobile data-storage applications in recent years has created a significant demand for high-capacity, fast-access, and low-power memory devices. In particular, mobile devices, such as personal digital assistants, digital cameras, MP3 players, and mobile phones, are in need of data-storage systems with such specifications. With existing nonvolatile memories, such as HDDs, ferroelectric random-access memory (FRAM), and Flash memory, approaching their physical limits, probe-based storage is viewed as a promising alternative for future mobile devices. It is also being investigated as a potential technology for archival data storage. This section presents an overview of the recent literature on probe-based data storage. 


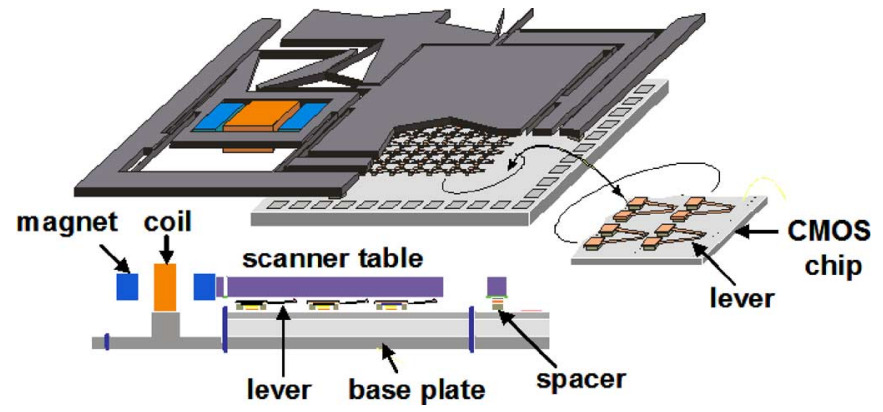

Fig. 23. Schematics of IBM's probe-based storage device prototype [214]

\section{A. Principle of Operation}

The concepts of data storage based on local probe techniques are derived from scanning probe microscopy, where nanometersharp tips are used to investigate and manipulate matter down to the atomic scale [209], [210]. Probe-storage technologies can be regarded as natural candidates for extending the areal density beyond that of conventional storage technologies. Early proposals to use a single atomic force microscope (AFM) tip in contact with a rotating polycarbonate substrate for writing and reading back topographic features date back to the early 1990s [211]-[213]. However, achieving high data rates represents a main challenge in building useful probe-storage devices, as the probes support data rates on the order of $100 \mathrm{~kb} / \mathrm{s}$. Thus, orders of magnitude increases in data rate are necessary for these devices to become competitive with conventional devices. A solution providing a substantial increase in data rate is obtained by employing MEMS-based arrays of probes that operate in parallel, with each probe performing write/read operations on an individual storage field. One implementation of a probe-based storage device where this concept was first applied and which is known in the scientific community as the "millipede" prototype developed by IBM, is presented in [12], [13], and [214], see Fig. 23. A generic probe-based storage system consists of a storage medium for recording digital information, a microscanner that moves the storage medium relative to an array of AFM-type probes with extremely sharp tips, a servo controller that controls movements of the microscanner during track-seek and track-following operations, and electronic circuits that implement, among other things, the necessary control and signal processing algorithms. The first fully functional probe-storage device prototype, including write/read operations by multiple probes in parallel and a closed-loop system for the nanopositioning of the scanner, was presented in [14], whereas the control strategies implemented in the prototype were presented in [16] and [17].

\section{B. Storage Medium}

Among the various probe-based data-storage concepts under investigation, thermomechanical recording and retrieval of data encoded as nanometer-scale indentations in thin polymer films is believed to be the most advanced [12]-[15]. In this method, the presence or absence of indentations corresponds to logical ones or zeros, respectively. Thermomechanical writing is achieved by applying a force through the microcantilever tip to the polymer layer and simultaneously heating the polymer layer locally. The written information is read by operating the microcantilever in contact mode and detecting the presence of an indentation using the thermal displacement sensor integrated into the microcantilever. Initial experimental results using single cantilevers have shown that data can be recorded at a density of $641 \mathrm{~Gb} / \mathrm{in}^{2}$ and read back with raw error rates better than $10^{-4}$ [215]. Later, a storage capacity of $840 \mathrm{~Gb} / \mathrm{in}^{2}$ using eight probes operating in parallel was demonstrated [14]. Furthermore, a feasibility study has shown that densities of 4 $\mathrm{Tb} / \mathrm{in}^{2}$ can be achieved by using an advanced polymer medium [216]. At the time of this writing, this is the only probe-storage technology that has reached this level of functionality.

A number of alternative storage media have been proposed for probe-based storage devices. A promising approach is based on phase-change media, where the memory application relies on a reversible phase transformation between the amorphous and the crystalline state [217]-[219]. In probe-storage applications, phase transformations are based on Joule heating induced by injecting a current pulse from the tip into the storage medium. The written information is read back by exploiting the different electrical resistivities of the two phases.

A group of LG researchers demonstrated the possibility of performing write/read/erase operations in a polymer medium without heating either the probe or the medium [220]. They observed that by applying pressure on a polystyrene-block-poly storage medium, microphase transitions occur that can be controlled to perform write, read or erase operations. A data-storage density of $1 \mathrm{~Tb} / \mathrm{in}^{2}$ has been demonstrated using this material. Two other nonvolatile storage methods studied are based on piezoelectric-mechanical [221] and ferroelectric storage media [222]-[225], whereby storage capacities of several $\mathrm{Tb} / \mathrm{in}^{2}$ with $\mathrm{read} / \mathrm{write}$ speed on the order of kilobits up to megabits per second per tip have been demonstrated.

\section{Probes}

As mentioned earlier, one drawback of probe-based data-storage is the relatively low data rate per microcantilever, e.g., about $100 \mathrm{~kb} / \mathrm{s}$ at acceptable power levels in thermomechanical-based systems. Hence, multiple microcantilevers must be operated in parallel to achieve data rates comparable to those of other data-storage devices. A probe array for the small-scale prototype system based on the thermomechanical probe-storage concept developed by IBM consists of an array of $4096(64 \times 64)$ microcantilevers with integrated read and write microheaters [226], see Fig. 24. Each microcantilever performs write/read/erase operations in an individual storage field approximately $100 \mu \mathrm{m} \times 100 \mu \mathrm{m}$ in size. The microcantilevers are actuated electrostatically, and are equipped with thermal sensors that can detect the probe distance from the storage medium. Competing systems are based on a very similar design, e.g., the device reported in [227] uses an array of 5000 electrostatically actuated microcantilevers spaced at $150 \mu \mathrm{m}$ pitch.

\section{Positioning System}

The positioning systems for scanning-probe-based data-storage device prototypes consist primarily of a microscanner that positions the storage medium relative to the 


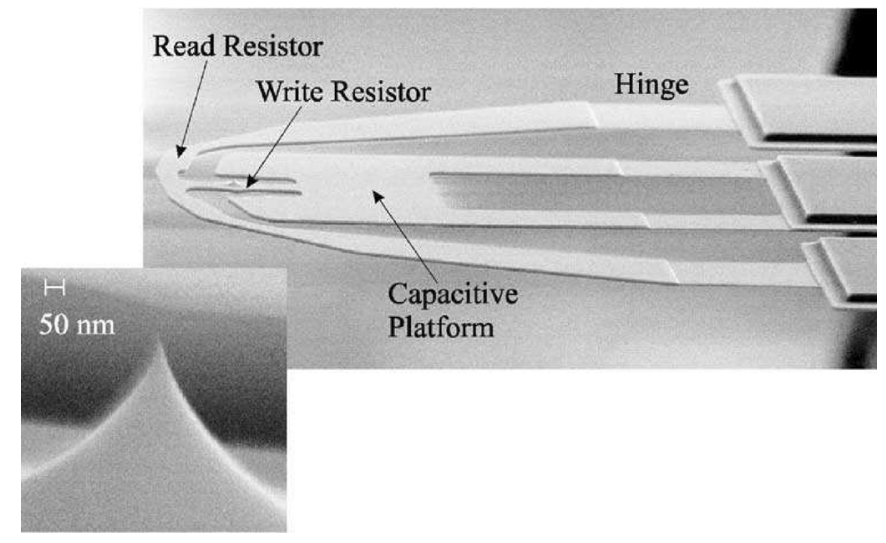

Fig. 24. Three-terminal integrated probe used in IBM's probe-storage device prototype. The inset shows an enlarged view of the tip, which is heated for write/ erase operations [215].

cantilever array, position sensors that provide global position information, and servo fields that generate medium-derived position information. These storage devices are required to have a small form factor so that they may be incorporated into mobile devices. This has led to the use of MEMS technology to realize the necessary positioning mechanisms. A typical system consists of a MEMS microscanner with $x-y$ motion capability and the storage medium spin-coated onto the scan table [12].

1) Actuation: The two most widely used actuation mechanisms in MEMS are electrostatic and electrothermal actuation. Piezoelectric, magnetic and shape memory alloy-based actuation methods have also been investigated. However, none are fully compatible with standard fabrication processes [228]-[230]. With some exceptions [231], [232], electromagnetic actuators are used in most of the prototype probe-based storage systems to move the scan table in the $x-y$ plane [227], [233], [234], [269]. Although this method of actuation is not compatible with available MEMS fabrication methods, it is chosen for its ability to generate a large stroke at reasonable power consumption.

In probe-based storage devices, data is stored in the storage fields along straight lines parallel to the $x$-axis, called tracks. Thus, to perform read/write/erase operations, the microscanner is moved in a zig-zag raster pattern. A key challenge is maintaining the position of the read/write probes on the target track as the probes are scanned along the length of the track during a $\mathrm{read} / \mathrm{write}$ operation. Each track is scanned in the horizontal or $x$-direction with constant velocity, while the fine positioning in the cross-track or $y$-direction is maintained in the presence of disturbances and noise. At the end of each track, a short seek operation is used to move into the next track if necessary. Precise positioning and navigation of the read/write transducers on the track center lines are critical in all types of storage devices. However, these requirements are much more stringent in probebased data-storage devices, in which accuracies on the order of a nanometer or less are needed to achieve reliable storage and retrieval of data [16], [17].

Recent interest has also focused on using non-raster-scan methods in AFM. In particular, two methods that have been

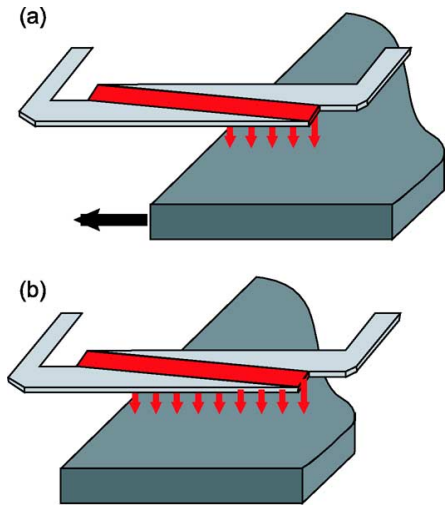

Fig. 25. Principle of operation of a MEMS thermal sensor [244].

proposed are based on tracking spiral [235], [236] and cycloid-like trajectories [237]. The main advantage of these methods is that for a mechanical scanner it is much easier to track a smooth trajectory, rather than a raster pattern with sharp corners. Thus, higher scan speeds can be achieved with the required positioning accuracy than with raster scans. The use of non-raster-scan methods in probe storage has been studied in [238].

2) Sensing: To sense the motion of the microscanner in both scan directions, position sensors are incorporated into MEMS microscanners. The most widely used displacement measurement technology in MEMS is capacitive sensing. The reasons are the simplicity of the sensor element itself, low power consumption, and good stability over temperature [229], [230]. Although many capacitive transducers have a nonlinear capacitance vs. displacement characteristic, feedback is commonly used to convert the signal to a linear output. This method has been used in two of the prototype probe-storage systems demonstrated, for which nanometer-level positioning accuracies have been reported [227], [239]. They have also been used in [232] where these electrostatic actuators are exploited for simultaneous sensing. An alternative approach to simultaneous sensing and actuation in electrostatic MEMS drives was first introduced in [240]. Similar methods had already been implemented in HDDs [241], [242], and in piezoelectrically actuated nanopositioners [243].

An innovative thermoelectric position-sensing method for MEMS-based probe-storage devices was reported in [244], see Fig. 25. In this approach, two pairs of thermoelectric position sensors are used to provide information on the $x-y$ position of the microscanner. The sensors are microheaters fabricated directly above the scan table such that they partially overlap with it. Displacement of the scan table results in a change of the overlapping area, which in turn results in a change of sensor temperature. The change in temperature translates into a change in current, which can be detected and used as a measure of the displacement. These thermal sensors operate over the entire travel range of the microscanner and hence are capable of providing global position information. The sensors are operated in differential mode, which results in a significant reduction in noise. Compared with capacitive sensors, they have a smaller form factor, which makes them a good proposition for use in MEMS devices. However, when operated at 
low frequencies, they are susceptible to flicker noise. Unlike in capacitive sensors, the low-frequency noise cannot be reduced by modulation/demodulation because it is an integral part of the sensor. Research efforts aimed at understanding the properties, limitations and other potential applications for thermoelectric sensing in MEMS are currently under way [245]-[250].

\section{E. Control Issues}

There are a number of control problems that naturally arise in probe-based storage systems. The purpose of this part is to present an overview of the issues and the solutions proposed in the recent literature.

1) Control of Self-Servo Write Process: Unlike most nanopositioning applications such as scanning probe microscopy, which require relative positioning [210], data-storage devices require absolute positioning. Moreover, this absolute positioning needs to be provided across the entire area of the storage field with nanometer-scale precision. This calls for inscription of position information on the medium. In the probe-storage device prototype reported in [13]-[16], a number of storage fields and their associated microcantilevers were reserved for the generation of position information. These storage fields are known as servo fields. Prewritten servo patterns in the servo fields can be used as an alternative position signal. This signal measures the deviation of the microcantilever tip from the track center during the read/write operation, and is essential in achieving repeatable positioning. The medium-derived PES is based on sequences of indentations (bursts), which are mutually displaced along the $y$-direction, arranged in such a way as to produce two signals in quadrature, which can be combined to provide a PES. The concept of medium-derived position sensing has been investigated by several other groups [231], [251], [252].

The complicating factor with generating the required servo fields is that the servo patterns have to be written using the global position sensors prior to the regular operation of the storage device by employing a self-servo write process. This is in contrast to HDDs, where the servo information is usually written with the assistance from an external positioning device [253]. Subnanometer-scale positioning accuracies are highly desirable while performing self-servo write operation, as the positioning errors incurred while writing the servo fields would appear as sensing noise while performing regular read/write operation of the device. Open-loop operation is not possible during self-servo write operation, primarily because of the lightly damped poles of the microscanner. Conventional feedback controllers, on the other hand, would make the system sensitive to measurement noise, particularly at low frequencies, and sensor drift.

To overcome this problem, the concept of shaping the noise sensitivity transfer function as applied to the self-servo write process was introduced in [254]. The underlying idea is based on the fact that when controlling a dynamical system, it is imperative to have frequency regions in which control is essential, e.g., the frequency regions close to the resonance frequency in the case of a nanopositioner. It is also evident that in these frequency regions, the closed-loop system would be sensitive to measurement noise. However, it is highly desirable, and possible, to carefully design the noise sensitivity transfer function such that the closed-loop system is insensitive to measurement noise outside these frequency regions. In nanopositioning applications that require positioning errors to be well below the resolution provided by the position sensors, a directed shaping of the noise sensitivity transfer function is essential. Control architectures for controlling the microscanner along both the $x$ and $y$-scan directions based on $H_{\infty}$ controllers [255] and resonant controllers were presented in [254]. Resonant controllers have been designed to augment damping of collocated highly resonant systems, and have been applied to flexible structures, with notable success [256], [257]. It was recently noted that resonant controllers, as well as other similar control structures such as positive position feedback control [258] and integral resonant control [259], belong to the class of strictly negative imaginary systems [260], which explains their remarkable robustness properties. Both control design methods studied in [254] were found to be capable of generating the desired noise sensitivity transfer functions. However, the resonant controller was found to be a more suitable choice for implementation because it could achieve the same objective with a simpler structure.

2) Track-Following Control: When a probe-storage device is in regular operation, the control system has two tasks. First, from an initial position, which could be quite arbitrary, the controller must locate the target track and position the scan table accordingly. This is achieved by the "seek and settle" procedure. A time-optimal controller was designed in [16], [261] to implement the seek operation. Furthermore, a worst-case seek time of about $1 \mathrm{~ms}$ has been reported for a probe-storage device [16], [261]. This is significantly faster than in other data-storage devices such as HDDs and ODDs, and is due to the small size of the MEMS-based storage devices and the fact that only quite tiny distances need to be travelled.

The second, and very critical, function of the servo system is to maintain the position of the read/write probes on the center of the target track during the scanning process along the length of this track. This is achieved by the "track-following" procedure. During track following, each track is scanned in the horizontal direction with constant velocity. This must be done while the fine positioning in the cross-track direction is maintained in the presence of disturbances and noise.

Although the dynamics of a microscanner along the $x$ - and $y$-scan directions are quite similar, the control design objectives are slightly different. Along the $x$-scan direction, a constant scan velocity, which is determined by the data rate of the storage device, needs to be maintained. Deviations from a uniform scan velocity during read and write operations result in timing jitter in the readback signals [262]. This phenomenon has a significant impact on the performance of the storage device. Low-frequency components of the positioning error are more tolerable thanks to timing-recovery circuits that form part of the read channel [18]. This implies that the $x$-axis control architecture can rely on the global position sensors alone, even though these sensors may be susceptible to drift and sensitivity variations [244]. LQG and PID controllers were designed and implemented on a prototype probe-storage device in [263]. This was 
followed by an LQG controller encompassing a feed-forward controller [16].

The $y$-axis control design problem is more involved. While scanning along the $x$-direction, the scanner must be maintained at a constant position in the $y$-direction such that the probes are close to the track center lines. Any deviation from the track center would lead to a loss in the signal strength during reading. This requires minimization of both the low- and the high-frequency components of the error signal. The medium-derived PES signal can be used as a secondary position sensor to complement the information derived from the global displacement sensors. While the latter generates good measurements at high frequencies, the former is a high-precision sensor at low frequencies. Thus, a controller can be designed to use the PES sensor at low and the global sensors at high frequencies. This idea of frequency separation was used in [264] to design a twoinput one-output $H_{\infty}$ controller that takes into account the noise spectral characteristics of the two sensors. An alternative approach reported in [17] involves an architecture similar to that of the $x$-direction, but with two tracking controllers, one for each of the two sensors. In this case, the tracking controllers are designed in such a way that frequency separation is enforced. It is worth observing that a similar situation arises, very naturally, in piezoelectric tube scanners, which has made it possible to use the concept of frequency separation for high-precision high-speed nanopositioning for AFM-type piezoelectric tube scanners [265], [266]. Two other track-following methods are proposed and discussed in [267].

Disturbance rejection is a key requirement in probe-storage control systems, particularly for track following. Disturbances can arise from a number of sources. They may be externally generated disturbances, such as vibration and noise, which are often modeled as input disturbances to the plant [254]. Or they may be due to nonlinear effects arising from the rotation of the scan table as it undergoes a raster scan [16]. Cross coupling between the slow and the fast axis of the scanner is also another unavoidable source of disturbance [17]. Also, simultaneous operation of a large number of microcantilevers during read or write operations can lead to a substantial disturbance [268]. All of these effects can be modeled as external disturbances. The frequency range over which they are substantial has to be identified, and their presence must be factored into the controller design.

\section{CONCLUSION}

In our information society, data storage plays a crucial role and is expected to assume ever increasing importance because of the exploding amount of data being generated and the need to preserve a large fraction of it. This paper reviewed key control technologies that allow storage systems based on HDDs, tape drives, and ODDs to continue to offer very large storage capacities in a cost-effective manner. The main challenges that control engineers will likely face in the foreseeable future have been addressed. Control-related research on emerging holographic data storage and probe-storage systems was also presented. Such applications promise to enable a dramatic increase in the achievable capacity of storage devices for archival systems and massive multimedia data storage.

\section{REFERENCES}

[1] "2010 digital universe study: A digital universe decade-Are you ready?" 2010. [Online]. Available: http://gigaom.files.wordpress.com/2010/05/2010-digital-universe-iview 5-4-1 0.pdf

[2] S. Devasia, E. Eleftheriou, and S. O. R. Moheimani, "A survey of control issues in nanopositioning," IEEE Trans. Control Syst. Technol., vol. 15 , no. 5, pp. 802-823, Sep. 2007.

[3] L.-S. Fan, H. H. Ottesen, T. Reiley, and R. Wood, "Magnetic recordinghead positioning at very high track densities using a microactuatorbased, two-stage servo system," IEEE Trans. Ind. Electron., vol. 42, no. 3, pp. 222-233, May 1995.

[4] S. Koganezawa, Y. Uematsu, T. Yamada, H. Nakano, J. Inoue, and T. Suzuki, "A dual-stage magnetic disk drive actuator using a piezoelectric device for a high track density," IEEE Trans. Magn., vol. 35, no. 2, pp. 988-992, Feb. 1999.

[5] M. C. Paek and K. Y. Kang, "Nanoprobe-based heating apparatus and heat-assisted magnetic recording head using the same," U.S. Patent Appl. 2009/0141387, Jun. 4, 2009.

[6] A. Argumedo, D. Berman, R. Biskeborn, G. Cherubini, R. D. Cideciyan, E. Eleftheriou, W. Häberle, D. Hellman, R. Hutchins, W. Imaino, J. Jelitto, K. Judd, P.-O. Jubert, M. Lantz, G. M. McClelland, T. Mittelholzer, S. Narayan, S. Ölçer, and P. Seger, "Scaling tape recording areal densities to $100 \mathrm{~Gb} / \mathrm{in}^{2}$," IBM J. Res. Develop., vol. 52 , no. $4 / 5$, pp. $513-527,2010$

[7] U. Boettcher, B. Raeymaekers, R. A. de Callafon, and F. E. Talke, "Dynamic modeling and control of a piezo-electric dual-stage tape servo actuator," IEEE Trans. Magn., vol. 45, no. 7, pp. 3017-3024, Jul. 2009.

[8] T. D. Milster, "Horizons for optical data storage," Opt. Photon. News, vol. 16, no. 3, pp. 28-33, Mar. 2005.

[9] J. Tominaga and T. Nakano, Optical Near-Field Recording. Berlin, Germany: Springer-Verlag, 2005.

[10] J. S. Baek, C. C. Chung, and M. Tomizuka, "Anti-shock controller design for optical disk drive systems with a nonlinear controller," in Proc. Amer. Control Conf., 2006, pp. 15-18.

[11] D. Psaltis and G. W. Burr, "Holographic data storage," Computer, vol. 31, no. 2, pp. 52-60, Feb. 1998.

[12] P. Vettiger, G. Cross, M. Despont, U. Drechsler, U. Dürig, B. Gotsmann, W. Häberle, M. A. Lantz, H. E. Rothuizen, R. Stutz, and G. K. Binnig, "The "millipede"-Nanotechnology entering data storage," IEEE Trans. Nanotechnol., vol. 1, no. 1, pp. 39-54, Jan. 2002.

[13] E. Eleftheriou, T. Antonakopoulos, G. K. Binnig, G. Cherubini, M. Despont, A. Dholakia, U. Dürig, M. A. Lantz, H. Pozidis, H. E. Rothuizen, and P. Vettiger, "Millipede-A MEMS-based scanning-probe data-storage system," IEEE Trans. Magn., vol. 39, no. 2, pp. 938-945, Feb. 2003.

[14] A. Pantazi, A. Sebastian, T. A. Antonakopoulos, P. Bächtold, A. R. Bonaccio, J. Bonan, G. Cherubini, M. Despont, R. A. DiPietro, U. Drechsler, U. Dürg, B. Gotsmann, W. Häberle, C. Hagleitner, J. L. Hedrick, D. Jubin, A. Knoll, M. A. Lantz, J. Pentarakis, H. Pozidis, R. C. Pratt, H. Rothuizen, R. Stutz, M. Varsamou, D. Weismann, and E. Eleftheriou, "Probe-based ultrahigh-density storage technology," IBM J. Res. Develop., vol. 52, no. 4-5, pp. 493-511, 2008.

[15] G. K. Binnig, G. Cherubini, M. Despont, U. Dürig, E. Eleftheriou, H. Pozidis, and P. Vettiger, "The "millipede"-A nanotechnology-based AFM data-storage system," in Springer Handbook of Nanotechnology, Part G Industrial Applications, B. Bhushan, Ed., 3rd ed. Berlin Heidelberg, Germany: Springer, 2010, ch. 45, pp. 1601-1632.

[16] A. Pantazi, A. Sebastian, G. Cherubini, M. Lantz, H. Pozidis, H Rothuizen, and E. Eleftheriou, "Control of MEMS-based scanning-probe data-storage devices," IEEE Trans. Control Syst. Technol., vol. 15, no. 5, pp. 824-841, Sep. 2007.

[17] A. Sebastian, A. Pantazi, H. Pozidis, and E. Eleftheriou, "Nanopositioning for probe-based data storage," IEEE Control Syst. Mag., vol. 28 , no. 4 , pp. 26-35, Jul. 2008

[18] H. Pozidis, G. Cherubini, A. Pantazi, A. Sebastian, and E. Eleftheriou, "Channel modeling and signal processing for probe storage channels," IEEE J. Sel. Areas in Commun., vol. 28, no. 2, pp. 143-157, Feb. 2010.

[19] J. P. Harris, W. B. Phillips, J. F. Wells, and W. D. Winger, "Innovations in the design of magnetic tape subsystems," IBM J. Res. Develop., vol. 25 , no. 5, pp. 691-699, 1981

[20] E. R. Childers, W. Imaino, J. H. Eaton, G. A. Jaquette, P. V. Koeppe, and D. J. Hellman, "Six orders of magnitude in linear tape technology: The one-terabyte project," IBM J. Res. Develop., vol. 47, no. 4, pp. 471-482, 2003.

[21] Data Interchange on $12.7 \mathrm{~mm}$ 384-Track Magnetic Tape Cartridges-Ultrium-1 Format, Standard ECMA-319, Jun. 2001. 
[22] G. A. Jaquette, "LTO: A better format for mid-range tape," IBMJ. Res. Develop., vol. 47, no. 4, pp. 429-444, 2003.

[23] D. B. Richards and M. P. Sharrock, "Key issues in the design of magnetic tapes for linear systems of high track density," IEEE Trans. Magn., vol. 34, no. 4, pp. 1878-1882, Apr. 1998.

[24] R. H. Dee, "Magnetic tape: The challenge of reaching hard-disk-drive data densities on flexible media," MRS Bulletin, vol. 31, no. 5, pp. 404-408, 2006.

[25] B. Steingrimsson and J. Moon, "Dropout compensation in magnetic tape channels by adaptive equalization and coding," IEEE Trans. Magn., vol. 37, no. 4, pp. 3981-3993, Apr. 2001.

[26] E. Eleftheriou, S. Ölçer, and R. A. Hutchins, "Adaptive noise-predictive maximum-likelihood (NPML) data detection for magnetic tape storage systems," IBM J. Res. Develop., vol. 54, no. 2, pp. 7:1-7:10, 2010.

[27] G. Cherubini, R. D. Cideciyan, L. Dellmann, E. Eleftheriou, W. Haeberle, J. Jelitto, V. Kartik, M. A. Lantz, S. Ölçer, A. Pantazi, H. E. Rothuizen, D. Berman, W. Imaino, P.-O. Jubert, G. McClelland, P. Koeppe, K. Tsuruta, T. Harasawa, Y. Murata, A. Musha, H. Noguchi, H. Ohtsu, O. Shimizu, and R. Suzuki, " $29.5 \mathrm{~Gb} / \mathrm{in}^{2}$ recording areal density on barium ferrite tape," IEEE Trans. Magn., vol. 47, no. 1, pp. 137-147, Jan. 2011.

[28] R. J. Taylor and F. E. Talke, "Investigation of roller interactions with flexible tape medium," Tribology Int., vol. 38, no. 6-7, pp. 599-605, 2005.

[29] B. Raeymaekers, R. J. Taylor, and F. E. Talke, "Correlation of lateral tape motion and tape tension transients," presented at the 15th Annu. ASME Inform. Storage Process. Syst. Conf., Santa Clara, CA, 2005.

[30] B. Raeymaekers and F. E. Talke, "Lateral motion of an axially moving tape on a cylindrical guide surface," J. Appl. Mechan., vol. 74, no. 5, pp. 1053-1056, 2007.

[31] B. Raeymaekers and F. E. Talke, "Measurements and sources of lateral tape motion: A review," J. Tribology, vol. 131, pp. 011 903-1-011 903-6, 2009

[32] J. M. Boyle, Jr. and B. Bushan, "Vibration response due to lateral tape motion and impulse force in a linear tape drive," Microsyst. Technol., vol. 11, pp. 48-73, 2005.

[33] S. Ölçer, E. Eleftheriou, and R. Hutchins, "Compensation of PLL loop delay in read channels for tape storage systems," presented at the IEEE Globecom, Honolulu, HI, 2009.

[34] R. C. Barrett, E. H. Klaassen, T. R. Albrecht, G. A. Jaquette, and J. H. Eaton, "Timing-based track-following servo for linear tape systems," IEEE Trans. Magn., vol. 34, no. 4, pp. 1872-1877, Apr. 1998.

[35] G. Cherubini, R. D. Cideciyan, E. Eleftheriou, and P. V. Koeppe, "Characterization of timing based servo signals," in Dig. IEEE Int. Magn. Conf. (INTERMAG), 2008, p. 600.

[36] G. Cherubini, R. D. Cideciyan, E. Eleftheriou, and J. Jelitto, "Characterization and detection of PPM-encoded servo signals in tape drives," in Proc. IEEE Pacific Rim Conf. Commun., Comput., Signal Process. (PacRim), 2009, pp. 342-347.

[37] G. Cherubini, E. Eleftheriou, J. Jelitto, and R. Hutchins, "Synchronous servo channel design for tape drive systems," in Proc. 17th Annu. ASME Inform. Storage Process. Syst. Conf., 2007, pp. 160-162.

[38] G. Cherubini and J. Jelitto, "LPOS symbol detection by soft-output combining of dual synchronous channels in tape drives," Microsyst. Technol., vol. 17, no. 5-7, pp. 857-862, 2011.

[39] G. F. Franklin, J. D. Powell, and M. Workman, Digital Control of Dynamic Systems, 3rd ed. Boston, MA: Addison-Wesley, 1997.

[40] G. E. Young and K. N. Reid, "Lateral and longitudinal dynamic behavior and control of moving webs," Trans. ASME: J. Dyn. Syst., Meas., Control, vol. 115(2b), pp. 309-317, 1993.

[41] J. J. Shelton and K. N. Reid, "Lateral dynamics of an idealized moving web," Trans. ASME: J. Dyn. Syst., Meas., Control, vol. 93, no. 3, pp. 187-192, 1971.

[42] P. D. Mathur and W. C. Messner, "Controller development for a prototype high-speed low-tension tape transport," IEEE Trans. Control Syst. Technol., vol. 6, no. 4, pp. 534-542, Jul. 1998.

[43] S. P. Panda and A. P. Engelmann, "Modeling and control system design of reel-to-reel tape drives," in Proc. Amer. Control Conf., 2002, pp. 927-932.

[44] M. B. Keshavan and J. A. Wickert, "Air entrainment during steadystate web winding," J. Appl. Mechan., vol. 64, no. 4, pp. 916-922, 1997.

[45] P. D. Mathur and W. C. Messner, "Frequency domain characterization of take-up reel air-entrainment in low tension and high-speed tape transport," Trans. ASME: J. Tribology, vol. 120, no. 3, pp. 554-558, 1997.
[46] M. B. Keshavan and J. A. Wickert, "Transient discharge of entrained air from a wound roll," J. Appl. Mechan., vol. 65, no. 4, pp. 804-810, 1998.

[47] Y. Lu and W. C. Messner, "Disturbance observer design for tape transport control," in Proc. Amer. Control Conf., 2001, pp. 2567-2571.

[48] Y. Lu and W. C. Messner, "Robust servo design for tape transport," in Proc. IEEE Int. Conf. Control Appl., 2001, pp. 1014-1019.

[49] M. D. Baumgart and L. Y. Pao, "Robust Lyapunov-based feedback control of nonlinear web-winding system," in Proc. 42nd IEEE Conf. Decision Control, 2003, pp. 6398-6405.

[50] M. D. Baumgart and L. Y. Pao, "Time-optimal control of web-winding systems with air entrainment," IEEE/ASME Trans. Mechatron., vol. 10, no. 3, pp. 257-262, Jun. 2005.

[51] K. C. Lin, "Frequency-domain design of tension observers and feedback controllers with compensation,", in Proc. 28th Annu. Conf. IEEE Ind. Electron. Soc. (IECON), 2002, pp. 1600-1605.

[52] K. C. Lin, "Observer-based tension feedback control with friction and inertia compensation," IEEE Trans. Control Syst. Technol., vol. 11, no. 1, pp. 109-118, Jan. 2003.

[53] M. D. Baumgart and L. Y. Pao, "Robust control of tape transport systems with no tension sensor," in Proc. 43rd IEEE Conf. Decision Control, 2004, pp. 4342-4349.

[54] H. Zhong, V. Kulkarni, and L. Pao, "Adaptive control for rejecting disturbances with time-varying frequencies in tape systems," in Proc. Amer. Control Conf., 2005, pp. 533-538.

[55] J. Holtz and L. Springob, "Identification and compensation of torque ripple in high-precision permanent magnet motor drives," IEEE Trans. Ind. Electron., vol. 43, no. 2, pp. 309-319, Mar. 1996.

[56] T. M. Jahns and W. L. Soong, "Pulsating torque minimization techniques for permanent magnet $\mathrm{AC}$ motor drives-A review," IEEE Trans. Ind. Electron., vol. 43, no. 2, pp. 321-330, Mar. 1996.

[57] L. Hoang, R. Perret, and R. Feuillet, "Minimization of torque ripples in brushless DC motor drives," IEEE Trans. Ind. Appl., vol. IA-22, no. 4, pp. 748-755, Jul. 1986.

[58] W.-C. Su, S. V. Drakunov, and U. Ozguner, "Sliding mode brushless DC motor current and torque control algorithms," in Proc. 1st IEEE Conf. Control Appl., 1992, pp. 910-915.

[59] H. Melkote and F. Khorrami, "Torque ripple attenuation for brushless DC motors via adaptive variable structure control," in Proc. 37th IEEE Conf. Decision Control, 1998, pp. 4640-4645.

[60] C. Lu, "Torque controller for brushless DC motors," IEEE Trans. Ind. Electron., vol. 46, no. 2, pp. 471-475, Mar. 1999.

[61] S. P. Panda and A. P. Engelmann, "Minimization of tape transient disturbances in track following tape drives with a disturbance observer," Microsyst. Technol., vol. 10, pp. 11-16, 2003.

[62] J. Jose, R. J. Taylor, R. A. de Callafon, and F. E. Talke, "Characterization of lateral tape motion and disturbances in the servo position error signal of a linear tape drive," Tribology Int., vol. 38, pp. 625-632, 2005.

[63] A. Pantazi, J. Jelitto, N. Bui, and E. Eleftheriou, "Track-follow control for tape storage," in Proc. IFAC Symp. Mechatron. Syst., 2010, pp. 532-537.

[64] C. E. Kinney and R. A. de Callafon, "A significant improvement to tape drive PES by canceling LTM with a robust, high performance controller," presented at the JSME-IIP/ASME-ISPS Joint Conf. Micromechatron. for Inform. Precision Equip., Ibaraki, Japan, 2009.

[65] R. A. de Callafon and C. E. Kinney, "Robust estimation and adaptive controller tuning for variance minimization in servo systems," $J$. $A d v$. Mechan. Design, Syst., Manuf., vol. 4, no. 1, pp. 130-142, 2010.

[66] A. Pantazi, M. Lantz, W. Häberle, W. Imaino, J. Jelitto, and E. Eleftheriou, "Active tape guiding," in Proc. 20th Annu. ASME Inform. Storage Process. Syst. Conf., 2010, pp. 304-306.

[67] M. Lantz, A. Pantazi, G. Cherubini, and J. Jelitto, "Nanoscale head positioning for flexible tape media," in Proc. 18th IFAC World Congr., 2011, pp. 869-874.

[68] V. Kartik, A. Pantazi, and M. Lantz, "High bandwidth track following for moving media," in Proc. 20th Annu. ASME Inform. Storage Process. Syst. Conf., 2010, pp. 265-267.

[69] B. Raeymaekers, M. R. Graham, R. A. de Callafon, and F. E. Talke, "Design of a dual-stage actuator tape head with high-bandwidth track following capability,” Microsyst. Technol., vol. 15, pp. 1525-1529, 2009.

[70] S. Schroeck, W. Messner, and R. McNab, "On compensator design for linear time-invariant dual-input single-output systems," IEEE/ASME Trans. Mechatron., vol. 6, no. 1, pp. 50-57, Mar. 2001

[71] C. F. Cutforth and L. Y. Pao, "Control using equal length shaped commands to reduce vibration," IEEE Trans. Control Syst. Technol., vol. 11 , no. 1 , pp. 62-72, Jan. 2003. 
[72] G. Cherubini and J. Jelitto, "Fast servo signal acquisition in tape drives," in Proc. IFAC Symp. Mechatron. Syst., 2010, pp. 538-544.

[73] K. Tsuruta, N. Bui, G. Cherubini, J. Jelitto, and A. Pantazi, "Head positioning with flangeless guide rollers in tape drives," in Proc. 21st Annu. ASME Inform. Storage Process. Syst. Conf., 2011, pp. 279-281.

[74] R. Fontana and S. Hetzler, "Millions of square inches (MSI) comparisons and implications for magnetic recording and solid state storage class memories," presented at the Dig. IEEE Int. Magn. Conf., Sacramento, CA, 2009.

[75] C. Du, L. Xie, J. N. Teoh, and G. Guo, " $H_{2}$ control for head positioning in axial and radial dimensions for self-servo track writing," IEEE Trans. Control Syst. Technol., vol. 16, no. 1, pp. 177-181, Jan. 2008.

[76] A. Sacks, "Position signal generation in magnetic disk drives," Ph.D. dissertation, Data Storage Syst. Center, Dept. Elect. Comput. Eng., Carnegie Mellon Univ., Pittsburgh, PA, 1995.

[77] J. Zhang, F. Hong, and S. Ge, "The strategy of designing seek current profile to reduce acoustic noise and residual vibrations," IEEE Trans. Magn., vol. 46, no. 6, pp. 1319-1322, Jun. 2010.

[78] C.-I. Kang, S.-E. Baek, and J.-S. Shim, "A new seek servo controller for minimizing power consumption in micro hard disk drives," IEEE Trans. Magn., vol. 40, no. 4, pp. 3127-3129, Apr. 2004.

[79] T. Semba and M. T. White, "Seek control to suppress vibrations of hard disk drives using adaptive filtering," IEEE/ASME Trans. Mechatron., vol. 13 , no. 5 , pp. $502-509$, Oct. 2008 .

[80] K. Eddy, J. Steele, and W. Messner, "Bias in disk drive rotary actuators: Characterization, prediction, and compensation," IEEE Trans. Magn., vol. 33, no. 3, pp. 3560-3565, May 1997.

[81] A. Okuyama, T. Yamaguchi, K. Shishida, and T. Horiguchi, "Reduction of acoustic noise in a head-positioning servo system of a magnetic disk drive by using initial value compensation and determining optimal mode switching conditions," Trans. Japan Soc. Mechan. Eng., vol. 68, no. 671, pp. 2016-2022, 2002.

[82] S.-H. Lee, S.-H. Chu, and C. C. Chung, "Analysis and design of servomechanism and its application to disk drives," IEEE Trans. Control Syst. Technol., vol. 11, no. 2, pp. 233-241, Mar. 2003.

[83] S. Baek, "Method, apparatus, and storage medium for controlling track seek servo in disk drive, and disk drive using same," U.S. Patent 7589931 B2, Feb. 9, 2010.

[84] M. Kobayashi and R. Horowitz, "Track seek control for hard disk dualstage servo systems," IEEE Trans. Magn., vol. 37, no. 2, pp. 949-954, Feb. 2001.

[85] S. Lee, Y.-H. Kim, and C. C. Chung, "Dual-stage actuator disk drives for improved servo performance: Track follow, track seek, and settle," IEEE Trans. Magn., vol. 37, no. 4, pp. 1887-1890, Apr. 2001.

[86] Y. Li, F. Marcass, R. Horowitz, R. Oboe, and R. Evans, "Robust dynamic modeling and control of dual-stage actuators," ASME J. Dyn. Syst., Meas., Control, vol. 128, no. 3, pp. 568-576, 2006.

[87] R. de Callafon, R. Nagamune, and R. Horowitz, "Robust dynamic modeling and control of dual-stage actuators," IEEE/ASME Trans. Magn,, vol. 42, no. 2, pp. 247-254, Feb. 2006.

[88] R. Conway, S. Felix, and R. Horowitz, "Model reduction and parametric uncertainty identification for robust $\mathrm{H}_{2}$ control synthesis for dual-stage hard disk drives," IEEE Trans. Magn., vol. 43, no. 9, pp. 3763-3768, Sep. 1999.

[89] J. Zheng, M. Fu, Y. Wang, and C. Du, "Nonlinear tracking control for a hard disk drive dual-stage actuator system," IEEE/ASME Trans. Mechatron., vol. 13, no. 5, pp. 510-518, Oct. 2008.

[90] Y. Huang, M. Banther, P. Mathur, and W. Messner, "Design and analysis of a high bandwidth disk drive servo system using an instrumented suspension," IEEE/ASME Trans. Mechatron., vol. 4, no. 2, pp. 196-206, Jun. 1999.

[91] S. Felix, J. Nie, and R. Horowitz, "Enhanced vibration suppression in hard disk drives using instrumented suspensions," IEEE Trans. Magn., vol. 45, no. 11, pp. 5118-5122, Nov. 2009.

[92] A. Jinzenji, T. Sasamoto, K. Aikawa, S. Yoshida, and K. Aruga, "Acceleration feedforward control against rotational disturbance in hard disk drives," IEEE Trans. Magn., vol. 37, no. 2, pp. 888-893, Feb. 2001.

[93] S. Hara, Y. Yamamoto, T. Omata, and M. Nakano, "Repetitive control system: A new type servo system for periodic exogenous signals," IEEE Trans. Autom. Control, vol. 33, no. 7, pp. 659-668, Jul. 1988.

[94] K. Chew and M. Tomizuka, "Digital control of repetitive errors in disk drive systems," IEEE Control Syst. Mag., vol. 10, no. 1, pp. 16-20, Jan. 1990.

[95] B. Francis and W. Wonham, "The internal model principle of control theory," Automatica, vol. 12, no. 5, pp. 457-465, 1976.
[96] C. Kempf, W. Messner, M. Tomizuka, and R. Horowitz, "Comparison of four discrete-time repetitive control algorithms," IEEE Control Syst., vol. 13, no. 6, pp. 48-54, Dec. 1993.

[97] M. Bodson, A. Sacks, and P. Khosla, "Harmonic generation in adaptive feedforward cancellation schemes," IEEE Trans. Autom. Control, vol. 39, no. 9, pp. 1939-1944, Sep. 1994.

[98] T. Atsumi, T. Okuyama, and M. Kobayashi, "Track-following control using resonant filter in hard disk drives," IEEE/ASME Trans. Mechatron., vol. 12, no. 4, pp. 472-479, Aug. 2007.

[99] H. Fujimoto, "RRO compensation of hard disk drives with multirate repetitive perfect tracking control," IEEE Trans. Ind. Electron., vol. 56, no. 10 , pp. 3825-3831, Oct. 2009.

[100] D. Shim, H. Lee, and L. Guo, "Mixed-objective optimization of a track-following controller using linear matrix inequalities," IEEE/ASME Trans. Mechatron., vol. 9, no. 4, pp. 636-643, Dec. 2004

[101] C. Du, L. Xie, J. Teoh, and G. Guo, "An improved mixed $\mathrm{H}_{2} / \mathrm{H}_{\infty}$ control design for hard disk drives," IEEE Trans. Control Syst. Technol., vol. 13 , no. 5, pp. 832-839, Sep. 2005

[102] X. Huang, R. Nagamune, and R. Horowitz, "A comparison of multirate robust track-following control synthesis techniques for dual-stage and multisensing servo systems in hard disk drives," IEEE Trans. Magn., vol. 42, no. 7, pp. 1896-1904, Jul. 2006.

[103] M. Graham, R. de Callafon, and L. Shrinkle, "Modeling and low-order control of hard disk drives with considerations for product variability," IEEE Trans. Magn., vol. 42, no. 10, pp. 2588-2590, Oct. 2006.

[104] G. Herrmann, B. Hredzak, M. Turner, I. Postlethwaite, and G. Guo, "Discrete robust anti-windup to improve a novel dual-stage large-span track-seek/following method," IEEE Trans. Control Syst. Technol., vol. 16, no. 6, pp. 1342-1351, Nov. 2008.

[105] R. Conway, J. Choi, R. Nagamune, and R. Horowitz, "Robust track-following controller design in hard disk drives based on parameter dependent Lyapunov functions," IEEE Trans. Magn., vol. 46, no. 4, pp. 1060-1068, Apr. 2010.

[106] Q. Hao, G. Guo, R. Chen, S. Chen, and T.-S. Low, "TMR online optimization using quasi-newton method for HDD servo systems," in Proc. Amer. Control Conf., 2000, pp. 3412-3416.

[107] M. Kobayashi, S. Nakagawa, T. Atsumi, and T. Yamaguchi, "Highbandwidth servo control designs for magnetic disk drives," in Proc. IEEE/ASME Int. Conf. Adv. Intell. Mechatron., 2001, pp. 1124-1129.

[108] W. Messner, M. Bedillion, L. Xia, and D. Karns, "Lead and lag compensators with complex poles and zeros design formulas for modeling and loop shaping," IEEE Control Syst. Mag., vol. 27, no. 1, pp. 44-54, Jan. 2007.

[109] W. Messner, "Classical control revisited: Variations on a theme," in Proc. 10th IEEE Int. Workshop Adv. Motion Control, 2008, pp. 15-20.

[110] M. Kobayashi, S. Nakagawa, and S. Nakamura, "A phase-stabilized servo controller for dual-stage actuators in hard disk drives," IEEE Trans. Magn., vol. 39, no. 2, pp. 844-850, Feb. 2003.

[111] D. Iamratanakul, H. Perez, and S. Devasia, "Feedforward trajectory design for output transitions in discrete-time systems: Disk-drive example," in Proc. Amer. Control Conf., 2003, pp. 3142-3147.

[112] A. Salton, Z. Chen, J. Zheng, and M. Fu, "Preview control of dual-stage actuator systems for superfast transition time," IEEE/ASME Trans. Mechatron., vol. 16, no. 4, pp. 758-763, Aug. 2011.

[113] M. H. Kryder and C. S. Kim, "After hard drives-What comes next?," IEEE Trans. Magn., vol. 45, no. 10, pp. 3406-3413, Oct. 2009.

[114] K. C. Pohlmann, The Compact Disc Handbook. Madison, WI: AR Editions, Inc., 1992.

[115] S. G. Stan, The CD-ROM Drive: A Brief System Description. Norwell, MA: Kluwer, 1998.

[116] D. Sarid and B. H. Schechtman, "A roadmap for optical data storage applications," Opt. Photon. News, vol. 18, no. 5, pp. 32-37, 2007.

[117] M. Steinbuch, P. J. M. van Groos, G. Schootstra, and O. H. Bosgra, "Multivariable control of a compact disc player using DSPs," in Proc. Amer. Control Conf., 1994, pp. 2434-2438.

[118] A. H. Chaghajerdi, "Sensing and control in optical drives," IEEE Control Syst. Mag., vol. 28, no. 3, pp. 23-29, Jun. 2008.

[119] T. H. M. Akkermans, "Digital control in optical disc drives," in Proc. Int. Conf. Consumer Electron., 2001, pp. 246-247.

[120] S. Bittanti, F. Dell'Orto, A. Di Carlo, and S. M. Savaresi, "Notch filtering and multirate control for radial tracking in high-speed DVDplayers," IEEE Trans. Consumer Electron., vol. 48, no. 1, pp. 56-62, Feb. 2002.

[121] T. Y. Doh, J. R. Ryoo, and M. J. Chung, "Design of a repetitive controller: An application to the track-following servo system of optical disk drives," IEE Proc. Control Theory Appl., vol. 153, no. 3, pp. 323-330, 2006. 
[122] G. Filardi, A. Besancon-Voda, and O. Sename, "Modeling identification and performance and analysis of a DVD player," in Proc. IEEE Int. Conf. Control Appl., 2002, pp. 623-628.

[123] K. M. Hock and W. Li, "Mathematical modeling of optical disc servo systems," in Conf. Dig. Opt. Data Storage Topical Meet., 2000, pp. $14-17$.

[124] K. Kinnstatter, M. Ojima, and S. Yonezawa, "Amplitude detection for the focus error in optical disks using a birefringent lens," Appl. Opt., vol. 29 , no. 29 , pp. $4408-4413,1990$.

[125] K. Nakai, H. Nakahara, D. Matsubara, T. Matozaki, N. Takeshita, T. Yoshihara, and K. Mori, "Focusing error detection using concentrically separated light beams for multilayer optical discs," Japan. J. Appl. Phys., vol. 45, no. 2B, pp. 1197-1203, 2006.

[126] S. L. DeVore, "Radial error signal simulation for optical disk drivers," Appl. Opt., vol. 25, pp. 4001-4006, 1986.

[127] C. Peng, W.-H. Yeh, and M. Mansuripur, "Measurements and simulations of differential phase-tracking signals in optical disk data storage," Appl. Opt., vol. 37, no. 20, pp. 4425-4432, Jul. 1998.

[128] $120 \mathrm{~mm}$ DVD—Read-Only Disk, 3rd Ed., Standard ECMA-267, Annex C, ECMA Technical Committee TC31, 2001.

[129] J. Shiraishi, T. Maruyama, Y. Takemoto, I. Ichimura, and S. Kobayashi, "New DPD tracking servo method by signal processing for high-density ROM discs," presented at the Optical Data Storage, OSA Tech. Dig. Series (CD) (Optical Society of America, 2007), 2007, paper TuB3, "," in

[130] K.-S. Kim, S.-H. Lee, and C. C. Chung, "A survey of control issues on optical data storage systems," in Proc. 18th IFAC World Congr., 2011, pp. 854-868.

[131] T. Ueyama, K. Sakai, and Y. Kurata, "A novel tracking servo system for multi-types of DVD with phase-shift DPP method," presented at the Int. Symp. Opt. Memory Opt. Data Storage Topical Meet., 2002, paper PD.1

[132] S. Lim and T.-Y. Jung, "Dynamics and robust control of a high speed optical pick up," J. Sound Vibr., vol. 221, no. 4, pp. 607-621, 1999.

[133] J.-D. Yang, L.-F. Pan, X.-D. Pei, and D.-Y. Xu, "Coupling enhanced control for optical disk drives," Japan. J. Appl. Phys., vol. 37, no. 4B, pp. 2203-2205, Apr. 1998.

[134] E. Yockyama, M. Nagasawa, and T. Katayama, "A disturbance suppression control system for car-mounted and portable optical disk drives," IEEE Trans. Consumer Electron., vol. 40, no. 2, pp. 92-99, May 1994

[135] P. F. Odgaard, J. Stoustrup, P. Andersen, M. V. Wickerhauser, and H. F. Mikkelsen, "A simulation model of focus and radial servos in compact disc players with disc surface defects," in Proc. IEEE Int. Conf. Control Appl., 2004, pp. 105-110.

[136] K.-S. Kim, "Analysis of tracking performance in optical data storage systems under eccentricity," in Proc. Amer. Control Conf., 2003, pp. 719-724.

[137] K.-S. Kim, "Analysis of optical data storage systems-Tracking performance with eccentricity," IEEE Trans. Ind. Electron., vol. 52, no. 4, pp. 1056-1062, Aug. 2005 .

[138] K.-S. Kim, "Eccentricity compensation in optical storage systems: Analysis and experiments," Japan. J. Appl. Phys., Pt. 1, vol. 41, no. 10, pp. 6302-6303, 2002

[139] K.-S. Kim and S.-P. Hong, "Enhancing the tracking performance in optical storage systems using a disturbance compensator," in Proc. Amer. Control Conf., 2002, pp. 1384-1388.

[140] H. Shim, H. Kim, and C. C. Chung, "Design of output regulator for rejecting periodic eccentricity disturbance in optical disc drive," in Proc. Int. Conf. Control, Autom., Syst., 2003, pp. 452-457.

[141] H. Shim, H. Kim, and C. C. Chung, "Design and experiment of add-on track following controller for optical disk drives based on robust output regulation," in Proc. Amer. Control Conf., 2004, pp. 1829-1835.

[142] M. Steinbuch, S. Weiland, and T. Singh, "Design of noise and periodtime robust high-order repetitive control, with application to optical storage," Automatica, vol. 43, no. 12, pp. 2086-2095, 2007.

[143] T. Y. Doh, J. R. Ryoo, and M. J. Chung, "Repetitive controller design for track-following servo system of an optical disk drive," in Proc. Int. Workshop Adv. Motion Control, 2002, pp. 176-181.

[144] F. Dong, Y. Wang, and J. Zhou, "Track following control design for odds by employing repetitive two-degree-of-freedom control scheme," IEEE Trans. Consumer Electron., vol. 49, no. 4, pp. 1186-1195, Nov. 2003.

[145] M. Heertjes and F. Sperling, "A nonlinear dynamic filter to improve disturbance rejection in optical storage drives," in Proc. IEEE Conf. Decision Control, 2003, pp. 3426-3430.
[146] K. Fujiyama, M. Tomizuka, and R. Katayama, "Digital tracking controller design for CD player using disturbance observer," in Proc. 5th IEEE Int. Workshop Adv. Motion Control, 1998, pp. 598-603.

[147] K. Ohishi, T. Miyazaki, and Y. Nakamura, "High performance ultra-low speed servo system based on doubly coprime factorization and instantaneous speed observer," IEEE/ASME Trans. Mechatron., vol. 1, no. 1, pp. 89-98, Mar. 1996.

[148] K. Arai, H. Okumura, H. Tokumaru, and K. Ohishi, "Improvement of performance of a tracking servo system for an optical disk drive," Japan. J. Appl. Phys., vol. 39, no. 2B, pp. 855-861, Feb. 2000.

[149] K. Zhou and J. C. Doyle, Essentials of Robust Control. Englewood Cliffs, NJ: Prentice-Hall, 1998.

[150] K. Ohishi, K. Kudo, Y. Hayakawa, H. Yanagisawa, D. Koide, and H. Tokumaru, "Robust feedforward tracking servo system for optical disk recoding system," in Proc. IECON, 2001, pp. 1710-1715.

[151] C. W. Lee and C. C. Chung, "Design of a new multi-loop disturbance observer for optical disk drive systems," IEEE Trans. Magn., vol. 45, no. 5, pp. 2224-2227, May 2009.

[152] J. R. Ryoo, T. Y. Doh, and M. J. Chung, "Robust disturbance observer for the track-following control system of an optical disk drive," Control Eng. Pract., vol. 12, pp. 577-585, 2004.

[153] K. Ohishi, T. Miyazaki, K. Inomata, H. Yanagisawa, D. Koide, and H. Tokumaru, "Robust tracking servo system considering force disturbance for the optical disk recording system," IEEE Trans. Ind. Electron., vol. 53, no. 3, pp. 838-847, Jun. 2006.

[154] J. R. Ryoo, K. Jin, J. H. Moon, and M. J. Chung, "Track-following control using a disturbance observer with asymptotic disturbance rejection in high-speed optical disk drives," IEEE Trans. Consumer Electron., vol. 49, no. 4, pp. 1178-1185, Nov. 2003.

[155] D. Koide, H. Tokumaru, K. Ohishi, T. Hayano, I. Shibutani, and T. Miyazaki, "High-speed tracking servo using zero phase error tracking-feed-forward method for professional-use optical disks over 10000 rpm," Japan. J. Appl. Phys., vol. 46, no. 6B, pp. 3765-3770, 2007

[156] Y. Q. Chen, K. L. Moore, J. Yu, and T. Zhang, "Iterative learning control and repetitive control in hard disk drive industry-A tutorial," Int. J. Adapt. Control Signal Process., vol. 22, no. 4, pp. 325-343, 2008.

[157] M. Steinbuch, "Repetitive control for systems with uncertain periodtime," Automatica, vol. 38, pp. 2103-2109, 2002.

[158] J. Leyva-Ramos, M. G. Ortiz-Lopez, and L. H. Diaz-Saldierna, "Disturbance rejection control scheme for optical disk drive systems," IEEE Trans. Magn., vol. 46, no. 10, pp. 3772-3777, Oct. 2010.

[159] D. A. Bristow, M. Tharayil, and A. G. Alleyne, "A survey of iterative learning control," IEEE Control Syst. Mag., vol. 26, no. 3, pp. 96-114, 2006.

[160] J. X. Xu, T. H. Lee, and H. W. Zhang, "Comparative studies on repeatable runout compensation using iterative learning control," in Proc. Amer. Control Conf., 2001, pp. 2834-2839.

[161] W. Kim, H. Kim, C. C. Chung, and M. Tomizuka, "Adaptive output regulation for the rejection of a periodic disturbance with an unknown frequency," IEEE Trans. Control Syst. Technol., vol. 19, no. 5, pp. 1296-1304, Sep. 2011.

[162] L. J. Brown and Q. Zhang, "Identification of periodic signals with uncertain frequency," IEEE Trans. Signal Process., vol. 51, no. 6, pp. 1538-1545, Jun. 2003.

[163] J.-J. Liu and Y.-P. Yang, "Frequency adaptive control technique for compact disk drives for rejecting periodic runout," Control Eng. Pract., vol. 12, pp. 31-40, 2004.

[164] J.-J. Liu and Y.-P. Yang, "Stability of the frequency adaptive control technique and its application to compact disk drives," Control Eng. Pract., vol. 13, no. 5, pp. 629-639, May 2005.

[165] T. Miyazaki, K. Ohishi, and D. Koide, "Robust feedforward tracking control based on sudden disturbance observer and ZPET control for optical disk recording system," in Proc. IEEE Int. Conf. Ind. Appl. Electron., Control, Robot., etc., 2004, pp. 353-358.

[166] Y. Zhou, M. Steinbuch, M. van der Aa, and H. Ladegaard, "Anti-shock controller design for optical drives," Control Eng. Pract., vol. 12, no. 7, pp. 811-817, Jul. 2004

[167] E. Vidal, P. Andersen, J. Stoustrup, and T. S. Pedersen, "A study on the surface defects of a compact disk," in Proc. IEEE Int. Conf. Control Appl., 2001, pp. 101-104.

[168] J. van Helvoirt, G. Leenknegt, M. Steinbuch, and H. J. Goosens, "Disc defect classification for optical disc drives," IEEE Trans. Consumer Electron., vol. 51, no. 3, pp. 856-863, Aug. 2005. 
[169] E. Vidal, K. G. Hansen, R. S. Andersen, K. B. Poulsen, J. Stoustrup, P. Andersen, and T. S. Pedersen, "Linear quadratic controller with fault detection in compact disk players," in Proc. IEEE Int. Conf. Control Appl., 2001, pp. 77-81.

[170] P. F. Odgaard, J. Stoustrup, P. Andersen, and H. F. Mikkelsen, "Estimating focus and radial distances, and fault residuals from CD player sensor signals by use of Kalman estimator," in Proc. IEEE Conf. Decision Control, 2003, pp. 1962-1967.

[171] M. Heertjes and M. Steinbuch, "Stability and performance of variable gain controller with application to a DVD storage drive," Automatica, vol. 40, no. 4, pp. 591-602, Apr. 2004.

[172] M. Heertjes, E. Pastink, N. van de Wouw, and H. Nijmeijer, "Experimental frequency-domain analysis of nonlinear controlled optical storage drives," IEEE Trans. Control Syst. Technol., vol. 14, no. 3, pp. 389-397, May 2006.

[173] M. Heertjes and G. Leenknegt, "Switching control in blu-ray disk drives," Mechatronics, vol. 20, pp. 453-463, 2010.

[174] J. Stoustrup, E. Vidal, P. Andersen, and T. S. Pedersen, "Current feedback for shock disturbance attenuation in a compact disc player," in Proc. IEEE Int. Conf. Control Appl., 2000, pp. 880-885.

[175] Y. Zhou, M. Steinbuch, and D. Kostic, "Estimator-based sliding mode control of an optical disc drive under shock and vibration,", in Proc. IEEE Int. Conf. Control Appl., 2002, pp. 631-636.

[176] T. D. Milster, "Near-field optics: A new tool for data storage," Proc. IEEE, vol. 88, no. 9, pp. 1480-1490, Sep. 2000.

[177] B. D. Terris, H. J. Mamin, and D. Rugar, "Near-field optical data storage," Appl. Phys. Lett., vol. 68, pp. 141-143, 1996.

[178] I. Ichimura, S. Hayashi, and G. S. Kino, "High-density optical recording using a solid immersion lens," Appl. Opt., vol. 36, no. 19, pp. 4339-4348, 1997.

[179] C. Peng, C. Mihalcea, D. Buchel, W. A. Challener, and E. C. Gage, "Near-field optical recording using a planar solid immersion mirror," Appl. Phys. Lett., vol. 87, p. 151105, 2005.

[180] S. M. Mansfield, W. R. Studenmund, G. S. Kino, and K. Osato, "Highnumerical-aperture lens system for an optical storage head," Opt. Lett., vol. 18 , no. 4 , p. $305,1993$.

[181] J. Tominaga, H. Fuji, A. Sato, T. Nakano, and N. Atoda, "The characteristics and the potential of super resolution near-field structure," Japan. J. Appl. Phys., vol. 39, no. 2B, pp. 957-961, 2000.

[182] T. Ishimoto, K. Saito, T. Kondo, A. Nakaoki, and M. Yamamoto, "Near-field readout system for a high-density optical ROM disc," in Proc. SPIE, 2002, vol. 4342, p. 294.

[183] T. Ishimoto, K. Saito, M. Shinoda, T. Kondo, A. Nakaoki, and M. Yamamoto, "Gap servo system for biaxial device using an optical gap signal in a near field readout system," Japan. J. Appl. Phys., vol. 42, pp. 2719-2724, 2003.

[184] F. Zijp, M. van der Mark, C. Verschuren, J. Lee, J. van den Eerenbeemd, P. Urbach, and M. van der Aa, "High-density near-field optical recording with a solid immersion lens, conventional actuator, and a robust air gap servo," IEEE Trans. Magn., vol. 41, no. 2, pp. 1042-1046, Feb. 2005.

[185] T. Ishimoto, K. Saito, M. Shinoda, T. Kondo, A. Nakaoki, and M. Yamamoto, "Gap servo system for a biaxial device using an optical gap signal in a near field readout system," Japan. J. Appl. Phys., vol. 42, no. 5A, pp. 2719-2724, May 2003.

[186] J. G. Kim, T. H. Kim, H. Choi, Y. J. Yoon, J. Jeong, N. C. Park, H. Yang, and Y. P. Park, "Improved air-gap control for SIL-based near-field recording system," IEEE Trans. Magn., vol. 43, no. 2, pp. 811-813, Feb. 2007.

[187] J. G. Kim, M. S. Kang, W. H. Shin, N. C. Park, H. S. Yang, and Y. P. Park, "Improved air gap controller for SIL based near-field recording servo system," presented at the Int. Symp. Opt. Memory Opt. Data Storage Topical, 2008, paper TuP30.

[188] J. I. Lee, M. van der Aa, C. Verschuren, F. Zijp, and M. van der Mark, "Development of an air gap servo system for high data transfer rate near field optical recording," Japan. J. Appl. Phys., vol. 44, no. 5A, pp. $3423-3426,2005$.

[189] J.-G. Kim, M.-S. Kang, T.-W. Kwon, J. Jeong, N.-C. Park, H.-S. Yang, and Y.-P. Park, "Improved gap control system using a disturbance observer for near-field recording," Japan. J. Appl. Phys., vol. 47, no. 7, pp. 5947-5952, 2008.

[190] J. Ashley, M.-P. Bernal, G. W. Burr, H. Coufal, H. Guenther, J. A. Hoffnagle, C. M. Jefferson, B. Marcus, R. M. Macfarlane, R. M. Shelby, and G. T. Sincerbox, "Holographic data storage technology," IBM J. Res. Develop., vol. 44, no. 3, pp. 341-368, 2000.

[191] H. J. Coufal, G. T. Sincerbox, and D. Psaltis, Holographic Data Storage. New York: Springer-Verlag, 2000.
[192] L. Hesselink, S. S. Orlov, and M. C. Bashaw, "Holographic data storage systems," Proc. IEEE, vol. 92, no. 8, pp. 1231-1280, Aug. 2004.

[193] K. Curtis, "Holographic Storage Tutorial," in ODS Conf., 2009. [Online]. Available: http://www.inphase-technologies.com/downloads/pdf/technology/HDSforODs2009.pdf

[194] A. Hoskins, M. Ayres, and K. Curtis, "Servo and drive control," in Holographic Data Storage: From Theory to Practical Systems, K. Curtis, L. Dhar, A. Hill, W. Wilson, and M. Ayres, Eds. Chichester, U.K.: Wiley, 2010.

[195] H. Horimai and X. Tan, "Holographic information storage system: Today and future," IEEE Trans. Magn., vol. 43, no. 2, pp. 943-947, Feb. 2007.

[196] G. Zhou, D. Psaltis, F. Mok, and A. Pu, "Method and system to align holographic images," U.S. Patent 5982 513, Nov. 9, 1999.

[197] C. W. Lee, B. S. Kwak, C. C. Chung, and M. Tomizuka, "Design of the tracking controller for holographic digital data storage," IEEE/ASME Trans. Mechatron., vol. 15, no. 2, pp. 242-252, Apr. 2010.

[198] N. Y. Kim, K. Jung, K. Kim, P. Yoon, J. Park, and J. Park, "A novel angle servo for holographic data storage system," in Proc. Opt. Data Storage, 2007, pp. 66 201-M1-66 201-M7.

[199] J. C. Bae, I. Kim, Y. Park, and T. Jeong, "Apparatus for recording/reproducing holographic data and method of adjusting position of recording layer," U.S. Patent Appl. 12/548 653, Aug. 27, 2009.

[200] K. Takasaki, H. Mori, S. Yamada, T. Hori, K. Hirooka, H. Okada, K. Tokuyama, M. Hara, S. Seko, A. Fukumoto, and K. Okada, "Development of a coaxial-type holographic disk system with a small drive," in Proc. Opt. Data Storage Top. Meet., 2009, pp. 110-112.

[201] V. P. Ostroverkhov, B. L. Lawrence, X. Shi, Z. Ren, and Z. Pan, "System and method for tracking in single-bit holographic data storage," U.S. Patent Appl. 2010/0165816 A1, Jul. 1, 2010.

[202] H. Horimai, "Optical information recording apparatus and optical information reproducing apparatus," U.S. Patent Appl 2005/0030875A1, Feb. 10, 2005.

[203] H. Horimai, X. Tan, and J. Li, "Collinear holography," Appl. Opt., vol. 44, no. 13, pp. 2575-2579, 2005.

[204] J. Yang, H. Yang, S. H. Kim, J. Y. Park, and Y. P. Park, "Design of tracking servo control system for holographic data storage," Microsyst. Technol., vol. 15, no. 10, pp. 1711-1717, 2009.

[205] Z. Göröcs, T. Sarkadi, G. Erdei, and P. Koppa, "Hologram positioning servo for phase-encoded holographic data storage systems," Appl. Opt., vol. 49, no. 4, pp. 611-618, 2010.

[206] H. Y. S. Li and D. Psaltis, "Alignment sensitivity of holographic threedimensional disks," J. Opt. Soc. Amer. A, vol. 12, no. 9, pp. 1902-1912, 1995.

[207] A. Hoskins, B. Sissom, and K. Curtis, "Tolerances of a page-based holographic data storage system," in Optical Data Storage, ser. OSA Tech. Dig. Series (CD). : Optical Society of America, 2007, p. WB2.

[208] S. H. Kim, J. H. Kim, J. Yang, H. Yang, J. Y. Park, and Y. P. Park, "Tilt detection and servo control method for the holographic data storage system," Microsyst. Technol., vol. 15, no. 10, pp. 1695-1700, 2009.

[209] G. Binnig, C. F. Quate, and C. Gerber, "Atomic force microscope," Phys. Rev. Lett., vol. 56, no. 9, pp. 930-933, 1986.

[210] E. Meyer, H. J. Hug, and R. Bennewitz, Scanning Probe Microscopy The Lab on a Tip. New York: Springer, 2004.

[211] H. J. Mamin and D. Rugar, "Thermomechanical writing with an atomic force microscope tip,” Appl. Phys. Lett., vol. 61, pp. 1003-1005, 1992.

[212] R. P. Ried, H. J. Mamin, B. D. Terris, L. S. Fan, and D. Rugar, "6-MHz $2-\mathrm{N} / \mathrm{m}$ piezoresistive atomic-force-microscope cantilevers with incisive tips," J. Microelectromech. Syst., vol. 6, pp. 294-302, 1997.

[213] B. D. Terris, S. A. Rishton, H. J. Mamin, R. P. Ried, and D. Rugar, "Atomic force microscope-based data storage: Track servo and wear study," Appl. Phys. A, vol. 66, pp. S809-S813, 1998.

[214] E. Eleftheriou, P. Bächtold, G. Cherubini, A. Dholakia, C. Hagleitner, T. Loeliger, A. Pantazi, H. Pozidis, T. Albrecht, G. Binnig, M. Despont, U. Drechsler, U. Dürig, B. Gotsmann, D. Jubin, W. Häberle, M. A. Lantz, H. Rothuizen, R. Stutz, P. Vettiger, and D. Wiesmann, "A nanotechnology-based approach to data storage," in Proc. 29th Int. Conf. Very Large Data Bases (VLDB), 2003, pp. 3-7.

[215] H. Pozidis, W. Häberle, D. Wiesmann, U. Drechsler, M. Despont, T. R. Albrecht, and E. Eleftheriou, "Demonstration of thermomechanical recording at $641 \mathrm{Gbit} / \mathrm{in}^{2}$," IEEE Trans. Magn., vol. 40, no. 4 II, pp. 2531-2536, Jul. 2004.

[216] D. Wiesmann, C. Rawlings, R. Vecchione, F. Porro, B. Gotsmann, A. Knoll, D. Pires, and U. Duerig, "Multi Tbit/in ${ }^{2}$ storage densities with thermomechanical probes," Nano Lett., vol. 9, no. 9, pp. 3171-3176, 2009. 
[217] S. Gidon, O. Lemonnier, B. Rolland, O. Bichet, C. Dressler, and Y. Samson, "Electrical probe storage using Joule heating in phase change media," Appl. Phys. Lett., vol. 85, no. 26, pp. 6392-6394, 2004.

[218] C. D. Wright, M. Armand, and M. M. Aziz, "Terabit-per-square-inch data storage using phase-change media and scanning electrical nanoprobes," IEEE Trans. Nanotechnol., vol. 5, no. 1, pp. 50-61, Jan. 2006.

[219] C. D. Wright, P. Shah, L. Wang, M. M. Aziz, A. Sebastian, and H. Pozidis, "Write strategies for multiterabit per square inch scanned-probe phase-change memories," Appl. Phys. Lett., vol. 97, 2010, Paper 173104.

[220] A. Jo, W. Joo, W.-H. Jin, H. Nam, and J. K. Kim, "Ultrahigh-density phase-change data storage without the use of heating," Nature Nanotechnol., vol. 4, no. 11, pp. 727-731, 2009.

[221] C. S. Lee, H.-J. Nam, Y.-S. Kim, W.-H. Jin, S.-M. Cho, and J.-U. Bu, "Microcantilevers integrated with heaters and piezoelectric detectors for nano data-storage application," Appl. Phys. Lett., vol. 83, no. 23 , pp. 4839-4841, 2003 .

[222] B. M. Kim, D. E. Adams, Q. Tran, Q. Ma, and V. Rao, "Scanning probe charge reading of ferroelectric domains," Appl. Phys. Lett., vol. 94, no. 6 , p. 063105,2009

[223] Y. Cho, K. Fujimoto, Y. Hiranaga, Y. Wagatsuma, A. Onoe, K. Terabe, and K. Kitamura, "Terabit/inch ${ }^{2}$ ferroelectric data storage using scanning nonlinear dielectric microscopy nanodomain engineering system," Nanotechnol., vol. 14, no. 6, pp. 637-642, 2003.

[224] B. M. Kim, D. E. Adams, G. Tchelepi, Q. Tran, Q. Ma, and V. Rao, "Scanning probe charge reading of ferroelectric polarization with nanoscale resolution," Nanotechnol.: Fabrication, Particles, Characterization, MEMS, Electron., Photon., vol. 1, pp. 289-290, 2009.

[225] M. G. Forrester, J. W. Ahner, M. D. Bedillion, C. Bedoya, D. G. Bolten, K.-C. Chang, G. de Gersem ans Shan Hu, E. C. Johns, M. Nassirou, J. Palmer, A. Roelofs, M. Siegert, S. Tamaru, V. Vaithyanathan, F. Zavaliche, T. Zhao, and Y. Zhao, "Charge-based scanning probe readback of nanometer-scale ferroelectric domain patterns at megahertz rates," Nanotechnol., vol. 20, no. 22, p. 225501, 2009.

[226] M. Despont, U. Drechsler, R. Yu, H. B. Pogge, and P. Vettiger, "Waferscale microdevice transfer/interconnect: Its application in an AFMbased data-storage system," J. Microelectromech. Syst., vol. 13, no. 6, pp. 895-901, 2004.

[227] J. Heck, D. Adams, N. Belov, T. A. Chou, B. Kim, K. Kornelsen, Q. Ma, V. Rao, S. Severi, D. Spicer, G. Tchelepi, and A. Witvrouw, "Ultrahigh density MEMS probe memory device," Microelectron. Eng., vol. 87, no. 5-8, pp. 1198-1203, 2010.

[228] G. T. A. Kovacs, Micromachined Transducers Sourcebook. New York: McGraw Hill, 1998.

[229] G. M. Rebeiz, RF MEMS: Theory, Desig and Technology. New York: Wiley, 2003

[230] S. D. Senturia, Microsystem Design. New York: Springer, 2001.

[231] P. G. Hartwell, R. G. Walmsley, D. J. Fasen, and S. Hoen, "Integrated position sensing for control of XY actuator," in Proc. IEEE Sensors, 2004, pp. 1407-1410.

[232] C. K. Pang, Y. Lu, C. Li, J. Chen, H. Zhu, J. Yang, J. Mou, G. Guo, B. M. Chen, and T. H. Lee, "Design, fabrication, sensor fusion, and control of a micro X-Y stage media platform for probe-based storage systems," Mechatronics, vol. 19, no. 7, pp. 1158-1168, 2009.

[233] M. A. Lantz, H. E. Rothuizen, U. Drechsler, W. Häberle, and M. Despont, "A vibration resistant nanopositioner for mobile parallel-probe storage applications," J. Microelectromech. Syst., vol. 16, no. 1, pp. 130-139, 2007.

[234] J. J. Choi, H. S. Park, K. Y. Kim, and J. U. Jeon, "Electromagnetic micro x-y stage for probe-based data storage," J. Semicond. Technol. Sci., vol. 1, no. 1, pp. 84-93, Mar. 2001.

[235] I. A. Mahmood and S. O. R. Moheimani, "Fast spiral-scan atomic force microscopy," Nanotechnol., vol. 20, p. 365503, 2009.

[236] I. A. Mahmood, S. O. R. Moheimani, and B. Bhikkaji, "A new scanning method for fast atomic force microscopy," IEEE Trans. Nanotechnol., vol. 10, no. 2, pp. 203-216, Mar. 2010.

[237] Y. K. Yong, S. O. R. Moheimani, and I. R. Petersen, "High-speed cycloid-scan atomic force microscopy," Nanotechnol., vol. 21, p. 365503, 2010.

[238] A. G. Kotsopoulos and T. A. Antonakopoulos, "Nanopositioning using the spiral of Archimedes: The probe-based storage case," Mechatronics, vol. 20, no. 2, pp. 273-280, 2010.

[239] J.-I. Lee, X. Huang, and P. B. Chu, "Nanoprecision MEMS capacitive sensor for linear and rotational positioning," J. Microelectromechan. Syst., vol. 18, no. 3, pp. 660-670, 2009.
[240] J. Dong and P. M. Ferreira, "Simultaneous actuation and displacement sensing for electrostatic drives," J. Micromechan. Microeng., vol. 18, no. 3, p. 035011, 2008.

[241] C. K. Pang, G. Guo, M. M. Chen, and L. T. Lee, "Nanoposition sensing and control in HDD dual-stage servo systems," in Proc. IEEE Int. Conf. Control Appl., 2004, pp. 551-556.

[242] H. Yamada, M. Sasaki, and Y. Nam, "Control of a micro-actuator for hard disk drives using self-sensing," in Proc. 8th IEEE Int. Workshop Adv. Motion Control, 2004, pp. 147-152.

[243] A. J. Fleming and S. O. R. Moheimani, "Sensorless vibration suppression and scan compensation for piezoelectric tube nanopositioners," IEEE Trans. Control Syst. Technol., vol. 14, no. 1, pp. 33-44, Jan. 2006.

[244] M. A. Lantz, G. K. Binnig, M. Despont, and U. Drechsler, "A micromechanical thermal displacement sensor with nanometre resolution," Nanotechnol., vol. 16, no. 8, pp. 1089-1094, 2005.

[245] U. Dürig, "Fundamentals of micromechanical thermoelectric sensors," J. Appl. Phys., vol. 98, no. 4, pp. 1-14, 2005.

[246] A. Sebastian and D. Wiesmann, "Modeling and experimental identification of silicon microheater dynamics: A systems approach," J. Microelectromechan. Syst., vol. 17, no. 4, pp. 911-920, 2008.

[247] H. Rothuizen, M. Despont, U. Drechsler, C. Hagleitner, A. Sebastian, and D. Wiesmann, "Design of power-optimized thermal cantilevers for scanning probe topography sensing," in Proc. IEEE 22nd Int. Conf. Micro Electro Mechan. Syst. (MEMS), 2009, pp. 603-606.

[248] J. Chow and Y. Lai, "Displacement sensing of a micro-electro-therma actuator using a monolithically integrated thermal sensor," Sensors Actuators, A: Phys., vol. 150, no. 1, pp. 137-143, 2009.

[249] K. J. Kim and W. P. King, "Thermal conduction between a heated microcantilever and a surrounding air environment," Appl. Thermal Eng., vol. 29, no. 8-9, pp. 1631-1641, 2009.

[250] Y. Zhu, A. Bazaei, S. O. R. Moheimani, and M. Yuce, "A micromachined nanopositioner with on-chip electrothermal actuation and sensing," IEEE Electron Device Lett., vol. 31, no. 10, pp. 1161-1163, Oct. 2010.

[251] C. W. Lee, H. J. Kang, C. C. Chung, and Y.-S. Kim, "New position error signal generation method for SPM based data storage system," Microsyst. Technol., vol. 15, no. 10-11, pp. 1663-1674, 2009.

[252] D.-K. Min and S. Hong, "Servo and tracking algorithm for a probe storage system," IEEE Trans. Magn., vol. 41, no. 2, pp. 855-859, Feb. 2005.

[253] A. Al Mamun, G. Guo, and B. Chao, Hard Disk Drive: Mechatronics and Control. Boca Raton, FL: CRC Press, 2006.

[254] A. Sebastian, A. Pantazi, S. O. Moheimani, H. Pozidis, and E. Eleftheriou, "Achieving subnanometer precision in a MEMS-based storage device during self-servo write process," IEEE Trans. Nanotechnol., vol. 7, no. 5, pp. 586-595, Sep. 2008.

[255] A. Skogestad and I. Postlewaite, Multivariable Feedback Control: Analysis and Design. New York: Wiley, 1996.

[256] D. Halim and S. O. R. Moheimani, "Spatial resonant control of flexible structures-Application to a piezoelectric laminate beam," IEEE Trans. Control Syst. Technol., vol. 9, no. 1, pp. 37-53, Jan. 2001.

[257] B. J. G. Vautier and S. O. R. Moheimani, "Charge-driven piezoelectric actuators for structural vibration control: Issues and implementation," Smart Mater. Structures, vol. 14, no. 4, pp. 575-586, 2005.

[258] S. O. R. Moheimani, B. J. G. Vautier, and B. Bhikkaji, "Experimental implementation of extended multivariable PPF control," IEEE Trans. Control Syst. Technol., vol. 14, no. 3, pp. 443-445, May 2006.

[259] B. Bhikkaji and S. O. R. Moheimani, "Integral resonant control of a piezoelectric tube actuator for fast nano-scale positioning," IEEE/ ASME Trans. Mechatron., vol. 13, no. 5, pp. 530-537, Oct. 2008.

[260] A. Lanzon and I. R. Petersen, "Stability robustness of a feedback interconnection of systems with negative imaginary frequency response," IEEE Trans. Autom. Control, vol. 53, no. 4, pp. 1042-1046, May 2008.

[261] A. Sebastian, A. Pantazi, G. Cherubini, M. Lantz, H. Rothuizen, H. Pozidis, and E. Eleftheriou, "Towards faster data access: Seek operations in MEMS-based storage devices," in Proc. IEEE Conf. Control Appl., 2006, pp. 283-288.

[262] A. Sebastian, A. Pantazi, and H. Pozidis, "Jitter investigation and performance evaluation of a small-scale probe storage device prototype," in Proc. GLOBECOM-IEEE Global Telecommun. Conf., 2007, pp. 288-293.

[263] A. Pantazi, M. A. Lantz, G. Cherubini, H. Pozidis, and E. Eleftheriou, "A servomechanism for a micro-electromechanical-system-based scanning-probe data storage device," Nanotechnol., vol. 15, no. 10, pp. S612-S621, 2004. 
[264] A. Pantazi, A. Sebastian, H. Pozidis, and E. Eleftheriou, "Two-sensorbased $H_{\infty}$ control for nanopositioning in probe storage," in Proc. IEEE Conf. Decision Control, 2005, pp. 1174-1179.

[265] A. J. Fleming, A. Wills, and S. O. R. Moheimani, "Sensor fusion for improved control of piezoelectric tube scanners," IEEE Trans. Control Syst. Technol., vol. 16, no. 6, pp. 1265-1276, Nov. 2008.

[266] I. A. Mahmood, S. O. R. Moheimani, and K. Liu, "Tracking control of a nanopositioner using complementary sensors," IEEE Trans. Nanotechnol., vol. 8, no. 1, pp. 55-65, Jan. 2009.

[267] C. W. Lee, H. J. Kang, C. C. Chung, and H.-J. Nam, "Seek and track-follow for scanning probe microscopy-based data storage," IEEE Trans. Magn., vol. 45, no. 10, pp. 3695-3698, Oct. 2009.

[268] N. Ramakrishnan and M. Bedillion, "A novel study of head motion hysteresis issues in contact probe recording systems," Microsyst. Technol., vol. 15, no. 4, pp. 595-606, 2009.

[269] Smart Structures Materials Conf. Newport Beach, CA, Mar. 2001.

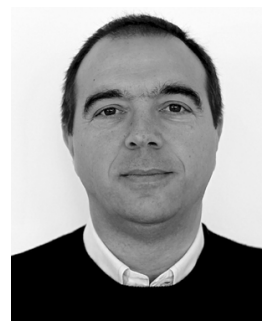

Giovanni Cherubini (S'80-M'82-SM'94-F'06) received a Laurea degree (summa cum laude) from the University of Padova, Padova, Italy, in 1981, and the M.S. and Ph.D. degrees from the University of California, San Diego, in 1984 and 1986, respectively, all in electrical engineering.

Since 1987, he has been with IBM ResearchZurich, Switzerland. His research interests comprise high-speed data-transmission, data-storage, and control systems. He was co-editor of the 100BASE-T2 Standard for Fast Ethernet transmission over voice-grade cables. More recently, he contributed to the realization of the first fully functional atomic-force-microscope-based data-storage prototype. He is currently focusing on servo-control technologies for tape drives. He holds over 40 patents in the areas of communications, data storage, and control systems, and was named Master Inventor at IBM in 2009.

Dr. Cherubini served as Editor of the IEEE TRANSACTIONS ON COMMUNICATIONS in the area of CDMA Systems, and is Associate Editor of the IEEE TRAnSACtions on CONTROL Systems TeChNOlogy. He was a co-recipient of the 2003 IEEE Communications Society Leonard G. Abraham Prize Paper Award, the 2009 IEEE Transactions on Control Systems Technology Outstanding Paper Award, and the 2009 IEEE CSS Control Systems Technology Award.

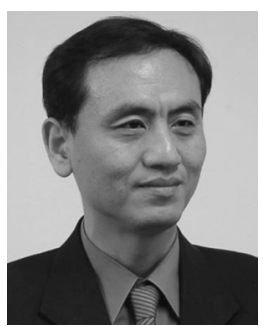

Chung Choo Chung (S'91-M'93) was born in Incheon, Korea. He received the B.S. and M.S. degrees in electrical engineering from Seoul National University, Seoul, Korea, and the Ph.D. degree in electrical and computer engineering from the University of Southern California, Los Angeles, in 1993.

From 1994 to 1997, he was with Samsung Advanced Institute of Technology, Korea, where he was a team leader responsible for hard disk drive servo system. In 1997, he joined the faculty of Hanyang University.

Dr. Chung was an Associate Editor for the Asian Journal of Control (AJC) from 2000 to 2002 and an Editor for the International Journal of Control, Automation and Systems (IJCAS) from 2003 to 2005. He was an Associate Editor for the 2003 IEEE Conference on Decision and Control (IEEE CDC), and an Associate Editor and the Co-Chair of Publicity of the International Federation of Automatic Control (IFAC) World Congress, Korea, in 2008. He served as a program co-chair of the International Conference on Control, Automation, and Systems (ICCAS) of the Society of Instrument and Control Engineers (SICE) 2009, Fukuoka, Japan. He was the Organizing Chair of ICCAS 2011. He served as a program committee member of the American Society of Mechanical Engineers (ASME) International Conference on Information Storage and Processing Systems (ISPS) 2011.

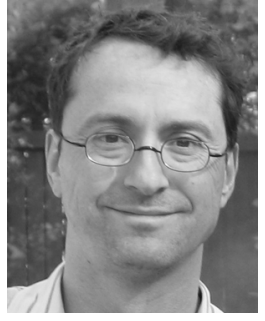

William C. Messner (SM'04) received the B.S. degree in mathematics from the Massachusetts Institute of Technology, Cambridge, MA, in 1985 and the M.S. and Ph.D. degrees in mechanical engineering from the University of California, Berkeley, in 1989 and 1992, respectively.

$\mathrm{He}$ is a Professor of mechanical engineering with courtesy appointments with the Electrical and Computer Engineering Department and The Robotics Institute, Carnegie Mellon University, Pittsburgh, PA. He specializes in the application of controls to data storage systems, robotic systems, and microfluidic systems for biological research. During his 2005-2006 sabbatical leave, he was a Visiting Professor of medicine at Harvard Medical School, Boston, MA, where he worked on the rheology protein networks.

Dr. Messner is a Fellow of the American Association for the Advancement of Science and a Fellow of the American Society of Mechanical Engineers.

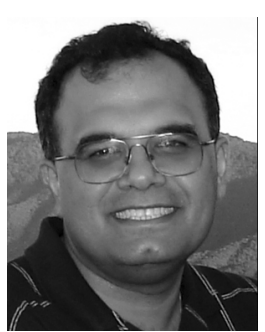

S. O. Reza Moheimani (F'11) joined The University of Newcastle, Australia, in 1997, where he founded and directs Laboratory for Dynamics and Control of Nanosystems, a multi-million-dollar state-of-the-art research facility dedicated to the advancement of nanotechnology through innovations in systems and control engineering. He is a Professor of Electrical Engineering and an Australian Research Council (ARC) Future Fellow. His current research interests are mainly in the area of dynamics and control at the nanometer scale, and include applications of control and estimation in nanopositioning systems for high-speed scanning-probe microscopy, modeling and control of microcantilever-based devices, control of microactuators in microelectromechanical systems (MEMS) and control issues related to ultrahigh-density probe-based data-storage systems.

Prof. Moheimani is a Fellow of IFAC and a Fellow of the Institute of Physics (U.K.). He was a co-recipient of the 2007 IEEE TRANSACTIONS ON CONTROL Systems Technology Outstanding Paper Award, and the 2009 IEEE CSS Control Systems Technology Award, together with a group of researchers from IBM Research-Zurich, where he has held several visiting appointments. He has served on the editorial board of a number of journals, including the IEEE TRansactions on CONTROL Systems TeChNOLOGY, the IEEE/ASME Transactions on Mechatronics, and Control Engineering Practice, and has chaired several international conferences and workshops. 